Algebraic 85 Geometric Topology

Volume 3 (2003) 623-675

Published: 25 June 2003

ATG

\title{
On the rho invariant for manifolds with boundary
}

\author{
PAUL KIRK \\ Matthias Lesch
}

\begin{abstract}
This article is a follow up of the previous article of the authors on the analytic surgery of $\eta$ - and $\rho$-invariants. We investigate in detail the (Atiyah-Patodi-Singer) $-\rho$-invariant for manifolds with boundary. First we generalize the cut-and-paste formula to arbitrary boundary conditions. A priori the $\rho$-invariant is an invariant of the Riemannian structure and a representation of the fundamental group. We show, however, that the dependence on the metric is only very mild: it is independent of the metric in the interior and the dependence on the metric on the boundary is only up to its pseudo-isotopy class. Furthermore, we show that this cannot be improved: we give explicit examples and a theoretical argument that different metrics on the boundary in general give rise to different $\rho$-invariants. Theoretically, this follows from an interpretation of the exponentiated $\rho-$ invariant as a covariantly constant section of a determinant bundle over a certain moduli space of flat connections and Riemannian metrics on the boundary. Finally we extend to manifolds with boundary the results of Farber-Levine-Weinberger concerning the homotopy invariance of the $\rho-$ invariant and spectral flow of the odd signature operator.
\end{abstract}

AMS Classification 58J28; 57M27, 58J32, 58J30

Keywords rho-invariant, eta-invariant

\section{Introduction}

The $\rho$-invariant of a closed odd-dimensional manifold was defined in [2] as a difference of two spectral invariants. To a closed Riemannian manifold $M$ and a unitary representation of its fundamental group $\alpha: \pi_{1}(M) \rightarrow U(n)$ Atiyah, Patodi, and Singer assigned the real number

$$
\rho(M, \alpha)=\eta\left(D_{\alpha}, M\right)-\eta\left(D_{\tau}, M\right)
$$

where $D_{\alpha}$ denotes the odd signature operator with coefficients in the flat bundle determined by $\alpha$, and $D_{\tau}$ is similar with respect to the trivial representation $\tau$, and $\eta(D)$ denotes the regularized signature of a self-adjoint Dirac operator $D$, introduced in [2]. As a consequence of their index theorem they showed that 
(1) $\rho(M, \alpha)$ is independent of the choice of Riemannian metric on $M$.

(2) $\rho(M, \alpha)$ extends the signature defect, that is, if $(M, \alpha)=\partial(W, \beta)$ for some manifold $W$ with unitary representation $\beta: \pi_{1}(W) \rightarrow U(n)$, then

$$
\rho(M, \alpha)=n \operatorname{Sign}(W)-\operatorname{Sign}_{\beta}(W)
$$

where $\operatorname{Sign}_{\beta}(W)$ denotes the signature of $W$ with local coefficients in the flat bundle determined by $\beta$.

Thus $\rho(M, \alpha)$ is a fundamental smooth invariant, but it remains largely mysterious since its general definition depends on the spectra of differential operators on $M$.

In [26] we defined the $\rho$-invariant $\rho(M, \alpha, g)$ in the case when the boundary of $M$ is non-empty and proved a non-additivity formula as a consequence of our cut-and-paste formula for $\eta$-invariants of Dirac operators: if $M$ is a closed manifold split into two parts $X$ and $Y$ along a hypersurface,

$$
\rho(M, \alpha)=\rho(X, \alpha, g)+\rho(Y, \alpha, g)+m\left(V_{X, \alpha}, V_{Y, \alpha}\right)_{(g, \alpha)}-m\left(V_{X, \tau}, V_{Y, \tau}\right)_{(g, \tau)} .
$$

(For the definitions of the terms see (2.4) and (2.8).)

It is the purpose of this article to explore the properties of $\rho(M, \alpha, g)$, particularly those which flow from this formula. We will describe the behavior of this invariant with respect to variations in Atiyah-Patodi-Singer (APS) boundary conditions, bordisms, and variations of $\alpha$ and $g$. As applications we prove generalizations of the main results of Farber-Levine-Weinberger [14] concerning the homotopy invariance of $\rho$ to manifolds with boundary. Special attention is given to the construction of explicit examples.

The invariant $\rho(M, \alpha, g)$ is defined as a difference of $\eta$-invariants for manifolds with boundary and as such is also a spectral invariant. This has the happy consequence that it is gauge and isometry invariant. But in contrast to the closed case, when the boundary of $M$ is non-empty the resulting invariant depends on the choice of Riemannian metric, $g$, on the boundary.

Hidden from the notation is the fact that elliptic boundary conditions are required to define $\eta$-invariants on manifolds with boundary. Our choice in [26] is to use APS boundary conditions with respect to the Lagrangian subspace of limiting values of extended $L^{2}$ solutions in the sense of [2]. This choice is intrinsic, homotopy invariant, and natural in a sense we will describe with respect to bordisms, but is not continuous in families. This fact is apparent when one considers families for which the dimension of the kernel of the tangential operator is not constant, but discontinuities can also occur in families for which the kernel is constant dimensional. 
For this reason it turns out to be useful to allow more general Lagrangian subspaces; we describe this generalization and derive the corresponding cut-andpaste formula for $\eta$ - and $\rho$-invariants with respect to arbitrary APS boundary conditions in Theorem 3.2. Among other things, Theorem 3.2 says:

Theorem Suppose that $M=Y \cup_{\Sigma} X, \alpha: \pi_{1}(M) \rightarrow U(n)$ is a representation, $W^{X}, W^{Y} \subset H^{*}\left(\Sigma ; \mathbb{C}_{\alpha}^{n}\right)$ are Lagrangian subspaces, and let $B$ be a flat connection on $M$ with holonomy $\alpha$ in cylindrical form near $\Sigma$. Then the difference $\eta\left(D_{B}, M\right)-\eta\left(D_{B, W^{X}}, X\right)-\eta\left(D_{B, W^{Y}}, Y\right)-m\left(W^{X}, W^{Y}\right)_{(\alpha, g)}$ equals the integer

$$
\tilde{\sigma}\left(V_{X, \alpha}, V_{Y, \alpha}, \gamma\left(W^{Y}\right)\right)-\tilde{\sigma}\left(\gamma\left(V_{X, \alpha}\right), W^{X}, W^{Y}\right) .
$$

In this statement $\eta\left(D_{B, W}, X\right)$ denotes the $\eta$-invariant of the odd signature operator coupled to a flat connection $B$ on the manifold $X$ with respect to APS boundary conditions determined by the Lagrangian subspace $W$ of the kernel of the tangential operator. Moreover, $m(V, W)_{(\alpha, g)}$ is an explicit realvalued invariant of pairs of Lagrangian subspaces of the Hermitian symplectic space $H^{*}\left(\Sigma ; \mathbb{C}_{\alpha}^{n}\right)$ with its induced $L^{2}$ metric (this is defined in Section 2), $\gamma$ is the associated complex structure, $\tilde{\sigma}$ is the Maslov triple index which appears in Wall's non-additivity theorem [36], $V_{X, \alpha}=\operatorname{image}\left(H^{*}\left(X ; \mathbb{C}_{\alpha}^{n}\right) \rightarrow H^{*}\left(\Sigma ; \mathbb{C}_{\alpha}^{n}\right)\right)$, and similarly for $V_{Y, \alpha}$.

This theorem generalizes [26, Theorem 8.8] to arbitrary APS boundary conditions. Taking differences gives a corresponding formula for $\rho$-invariants.

We next give a topological description of how the spaces $V_{X, \alpha}$ propagate across a bordism; the result is given as Theorem 4.1 which gives a functorial framework to keep track of APS boundary conditions and a companion additivity formula for $\eta$ and $\rho$.

With these technical results in place, we can then begin a careful investigation of how the $\rho$-invariants for manifolds with boundary depend on the choice of metric on the boundary and the representation. For example, we show:

Corollary 5.2 The $\rho$-invariant for a manifold with boundary depends on the Riemannian metric on the boundary only up to its pseudo-isotopy class. Precisely, if $f_{0}, f_{1}: \partial X \rightarrow \partial X$ are pseudo-isotopic diffeomorphisms, then

$$
\rho\left(X, \alpha, f_{0}^{*}(g)\right)=\rho\left(X, \alpha, f_{1}^{*}(g)\right) .
$$

In Section 6 we give explicit examples which show that $\rho(X, \alpha, g)$ and $m(V, W)$ depend on the choice of Riemannian metric: 


\section{Theorem 6.1}

(1) There exists a 3-manifold $Y$ with non-empty boundary, Riemannian metrics $g_{0}, g_{1}$ on $\partial Y$, and a representation $\alpha: \pi_{1} Y \rightarrow U(2)$ so that

$$
\rho\left(Y, \alpha, g_{0}\right) \neq \rho\left(Y, \alpha, g_{1}\right) .
$$

Examples exist with vanishing kernel of the tangential operator, i.e.

$$
\text { ker } A_{b} \cong H^{*}\left(\partial Y ; \mathbb{C}_{\alpha}^{2}\right)=0 \text {. }
$$

(2) There exist metrics $g_{0}$ and $g_{1}$ on the torus $T$ and 3-manifolds $X$ and $Y$ with boundary $T$ such that setting $V_{X}=\operatorname{image}\left(H^{*}(X ; \mathbb{C}) \rightarrow H^{*}(T ; \mathbb{C})\right)$ and $V_{Y}=$ image $\left(H^{*}(Y ; \mathbb{C}) \rightarrow H^{*}(T ; \mathbb{C})\right.$ ) (with $\theta$ the trivial conection),

$$
m\left(V_{X}, V_{Y}\right)_{\left(\theta, g_{0}\right)} \neq m\left(V_{X}, V_{Y}\right)_{\left(\theta, g_{1}\right)} .
$$

This theorem drives home the point that the choice of Riemannian metric on the boundary is an essential ingredient of the $\rho$-invariant on a manifold with non-empty boundary.

In Section 7 we extend to manifolds with boundary the results of Farber-LevineWeinberger concerning the homotopy invariance of the $\rho$-invariants and spectral flow of the odd signature operator. Let

$$
\chi\left(\pi_{1} X, U(n)\right)=\operatorname{Hom}\left(\pi_{1} X, U(n)\right) / \text { conjugation }
$$

and let $\mathscr{M}_{\partial X}$ denote the space of Riemannian metrics on $\partial X$. Notice that a map $F: X \rightarrow X^{\prime}$ which restricts to a diffeomorphism on the boundary and which induces an isomorphism on fundamental groups provides an identification of $\mathscr{M}_{\partial X}$ with $\mathscr{M}_{\partial X^{\prime}}$ and $\chi\left(\pi_{1} X, U(n)\right)$ with $\chi\left(\pi_{1} X^{\prime}, U(n)\right)$.

Theorem 7.2 Let $F: X \rightarrow X^{\prime}$ be a homotopy equivalence of compact manifolds which restricts to a diffeomorphism on the boundary. Then the difference

$$
\rho(X)-\rho\left(X^{\prime}\right): \chi\left(\pi_{1} X, U(n)\right) \times \mathscr{M}_{\partial X} \rightarrow \mathbb{R}
$$

factors through $\pi_{0}\left(\chi\left(\pi_{1} X, U(n)\right)\right) \times\left(\mathscr{M}_{\partial X} / \mathscr{D}_{\partial X}^{0}\right)$ (where $\mathscr{D}_{\partial X}^{0}$ denotes the group of diffeomorphisms of $\partial X$ pseudo-isotopic to the identity) and takes values in the rational numbers.

In other words there is a commutative diagram

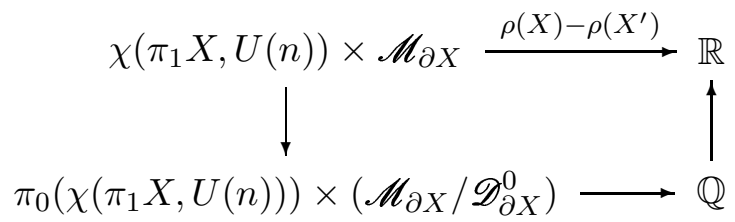

Moreover the difference $\rho(X, \alpha, g)-\rho\left(X^{\prime}, \alpha, g\right)$ vanishes for $\alpha$ in the path component of the trivial representation. 
We also show that the spectral flow of the odd signature operator coupled to a path of flat connections is a homotopy invariant. In the following statement $P(t)$ denotes a smooth path of self-adjoint APS boundary conditions with prescribed endpoints. (We show how to construct such a path in Lemma 7.3.)

Theorem 7.4 Suppose that $F: X^{\prime} \rightarrow X$ is a homotopy equivalence which restricts to a diffeomorphism $f=\left.F\right|_{\partial X^{\prime}}: \partial X^{\prime} \rightarrow \partial X$. Assume that $B_{t}$ is a continuous, piecewise smooth path of flat $U(n)$ connections on $E \rightarrow X$. Use $F$ to pull back the path $B_{t}$ to a path of flat connections $B_{t}^{\prime}$ on $X^{\prime}$ and to identify $\partial X$ with $\partial X^{\prime}$, and choose a path $P(t)$ of APS boundary conditions as in Lemma 7.3.

Then

$$
\mathrm{SF}\left(X, D_{B_{t}, P(t)}\right)_{t \in[0,1]}=\mathrm{SF}\left(X^{\prime}, D_{B_{t}^{\prime}, P(t)}\right)_{t \in[0,1]} .
$$

In Section 8 we use the machinery of determinant bundles, especially the DaiFreed theorem, to study the variation of $\rho(X, \alpha, g)$ modulo $\mathbb{Z}$. By working modulo $\mathbb{Z}$ one loses geometric information but the discontinuities of $\rho$ as a function of $\alpha$ are eliminated. In particular variational techniques can be applied.

Theorem 8.4 implies the following. In this theorem $\nabla^{Q}$ denotes the connection on the determinant bundle as introduced by Quillen in [32]. (See [5] for the construction of $\nabla^{Q}$ in general.)

Theorem The assignment of the exponentiated $\rho$-invariant to a flat $S U(n)$ connection $B$ on a manifold with boundary $X$ and a choice of Riemannian metric $g$ on $\partial X$,

$$
(B, g) \mapsto \exp (\pi i \rho(X, \alpha, g)),
$$

(where $\alpha$ is the holonomy of $B$ ) defines a smooth horizontal (with respect to the connection $\nabla^{Q}$ ) cross section of the determinant bundle of the family of tangential operators to the odd signature operators.

This theorem allows one to relate the $\bmod \mathbb{Z}$ reduction of the $\rho$-invariant on manifolds with isomorphic fundamental groups and diffeomorphic boundaries, and also shows that the manner in which $\rho(X, \alpha, g)$ depends on the choice of metric $g$ on $\partial X$ is intimately tied to the connection $\nabla^{Q}$.

For example, the following is a consequence of Theorem 8.5. We view $\rho(X)$ as a function of the conjugacy class of the representation $\alpha$ and the metric $g$. 
Theorem Let $X$ and $X^{\prime}$ be two odd dimensional manifolds and suppose that $F: X^{\prime} \rightarrow X$ is a smooth map which induces an isomorphism on fundamental groups and such that the restriction $f=\left.F\right|_{\partial X^{\prime}}: \partial X^{\prime} \rightarrow \partial X$ is a diffeomorphism.

Then there is a factorization

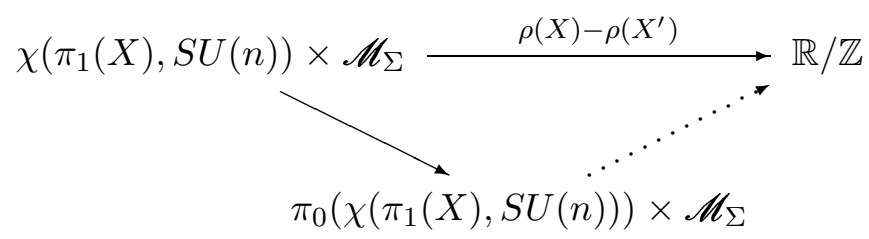

and $\rho(X)-\rho\left(X^{\prime}\right)$ is zero on the path component of the trivial representation. The result holds for $U(n)$ replacing $S U(n)$ if $\operatorname{dim} X=4 \ell-1$.

These results, together with the cut-and-paste formula for $\rho$-invariants (Theorem 3.2) are a step in the program of determining what the homotopy properties of the $\rho$-invariant are. A discussion of problems in this topic is given in Section 9 , including the following consequence of Theorem 9.2 concerning the homotopy invariance of the $\rho$-invariant for closed manifolds.

Theorem Let $M$ and $M^{\prime}$ be closed manifolds, and suppose there exists a separating hypersurface $\Sigma \subset M$ and a smooth homotopy equivalence $F: M^{\prime} \rightarrow M$ so that the restriction of $F$ to $F^{-1}(\Sigma)$ is a diffeomorphism. Write $M=X \cup_{\Sigma} Y$ and $M^{\prime}=X^{\prime} \cup_{\Sigma} Y^{\prime}$ and suppose that $F$ restricts to homotopy equivalences $X^{\prime} \rightarrow X$ and $Y^{\prime} \rightarrow Y$. Let $\alpha \in \chi\left(\pi_{1} M, U(n)\right)$.

If the restriction $\left.\alpha\right|_{X}$ (resp. $\left.\alpha\right|_{Y}$ ) of $\alpha$ to $\pi_{1} X$ (resp. $\pi_{1} Y$ ) lies in the path component of the trivial representation of $\chi\left(\pi_{1} X, U(n)\right)$ (resp. $\left.\chi\left(\pi_{1} Y, U(n)\right)\right)$ then $\rho(M, \alpha)=\rho\left(M^{\prime}, \alpha\right)$.

We finish the article with a brief discussion of the relation of our investigations to one of the approaches to the program of constructing topological quantum field theories proposed in [1].

\section{Acknowledgements}

The authors thank J.F. Davis, C. Livingston, S. Paycha, and K.P. Wojciechowski for very helpful discussions.

The first named author gratefully acknowledges the support of the National Science Foundation under grant no. DMS-0202148. 


\section{The $\rho$-invariant on manifolds with boundary}

We begin by recalling the context and the definition of the $\rho$-invariant for a manifold with boundary. More details can be found in [26].

Let $X$ be a $(2 k+1)$-dimensional smooth, oriented, compact manifold with (possibly empty) boundary $\Sigma$. Fix a Riemannian metric $\tilde{g}$ on $X$ in product form near the boundary $\Sigma$. To keep track of signs it is crucial to fix a convention for the orientation of a collar of the boundary. In this paper we will use the convention of [26]: if not indicated otherwise a collar of the boundary will be written as $\Sigma \times[0, \epsilon)$, i.e. the manifold $X$ is "on the right" of the boundary. The choice of the sign convention has consequences for the definition of $A_{b}$ and $\gamma$ (and hence the Hermitian symplectic structure on $H^{*}\left(\Sigma ; \mathbb{C}_{\alpha}^{n}\right)$ ) below.

Let $B$ be a flat $U(n)$ connection on $X$ in product form near the boundary, i.e. $\left.B\right|_{\Sigma \times[0, \epsilon)}=\pi^{*}(b)$ for some flat connection $b$ on $\Sigma$; here $\pi: \Sigma \times[0, \epsilon) \rightarrow \Sigma$ denotes the projection. Denote by $\alpha: \pi_{1} X \rightarrow U(n)$ the holonomy representation of $B$. Since it will be central in what follows, denote the restriction of $\tilde{g}$ to the boundary $\Sigma$ by $g$.

The odd signature operator coupled to the flat connection $B$

$$
D_{B}: \oplus_{p} \Omega^{2 p}(X ; E) \rightarrow \oplus_{p} \Omega^{2 p}(X ; E)
$$

is defined by

$$
D_{B}(\beta)=i^{k+1}(-1)^{p-1}\left(* d_{B}-d_{B} *\right)(\beta) \text { for } \beta \in \Omega^{2 p}(X ; E) .
$$

Here, $*: \Omega^{\ell}(X ; E) \rightarrow \Omega^{2 k+1-\ell}(X ; E)$ denotes the Hodge ${ }^{*}$ operator (which is determined by the Riemannian metric $\tilde{g}$ on $X), d_{B}: \Omega^{\ell}(X ; E) \rightarrow \Omega^{\ell+1}(X ; E)$ denotes the covariant derivative associated to the flat connection $B$, and $E \rightarrow$ $X$ denotes the associated Hermitian $\mathbb{C}^{n}$ vector bundle.

On the collar $\Sigma \times[0, \epsilon), D_{B}$ takes the form (after conjugating with a certain unitary transformation, see $[26,(8.1)]$ for details)

$$
D_{B}=\gamma\left(\frac{\partial}{\partial x}+A_{b}\right)
$$

where the de Rham operator

$$
A_{b}: \oplus_{k} \Omega^{k}\left(\Sigma ;\left.E\right|_{\Sigma}\right) \rightarrow \oplus_{k} \Omega^{k}\left(\Sigma ;\left.E\right|_{\Sigma}\right)
$$

is defined by

$$
A_{b}(\beta)=\left\{\begin{aligned}
-\left(d_{b} \hat{*}+\hat{*} d_{b}\right) \beta, & \text { if } \beta \in \oplus_{k} \Omega^{2 k}\left(\Sigma ;\left.E\right|_{\Sigma}\right), \\
\left(d_{b} \hat{*}+\hat{*} d_{b}\right) \beta, & \text { if } \beta \in \oplus_{k} \Omega^{2 k+1}\left(\Sigma ;\left.E\right|_{\Sigma}\right) .
\end{aligned}\right.
$$

Algebraic 83 Geometric Topology, Volume 3 (2003) 
In these formulas $\hat{*}$ denotes the Hodge $*$ operator on $\Sigma$ and

$$
\gamma: \oplus_{p} \Omega^{p}\left(\Sigma ;\left.E\right|_{\Sigma}\right) \rightarrow \oplus_{p} \Omega^{p}\left(\Sigma ;\left.E\right|_{\Sigma}\right)
$$

coincides with $\hat{*}$ up to a constant:

$$
\gamma(\beta)= \begin{cases}i^{k+1}(-1)^{p-1} \hat{*} \beta, & \text { if } \beta \in \Omega^{2 p}\left(\Sigma ;\left.E\right|_{\Sigma}\right), \\ i^{k+1}(-1)^{k-q_{\hat{*}}} \beta, & \text { if } \beta \in \Omega^{2 q+1}\left(\Sigma ;\left.E\right|_{\Sigma}\right) .\end{cases}
$$

One calculates that $\gamma^{2}=-I d, \gamma A_{b}=-A_{b} \gamma$, and that $\gamma$ is unitary with respect to the $L^{2}$ inner product on $\Omega^{*}\left(\Sigma ;\left.E\right|_{\Sigma}\right)$ defined by

$$
\left\langle\beta_{1}, \beta_{2}\right\rangle=\int_{\Sigma} \beta_{1} \wedge \hat{*} \beta_{2} .
$$

(The Riemannian metric on $\Sigma$ is used to define the Hodge $*$-operator $\hat{*}$, and we have suppressed the notation for the inner product in the bundle $E$.) The operator $A_{b}$ is elliptic and self-adjoint and hence one has an orthogonal decomposition

$$
L^{2}\left(\Omega^{*}\left(\Sigma ;\left.E\right|_{\Sigma}\right)\right)=F_{b}^{-} \oplus \operatorname{ker} A_{b} \oplus F_{b}^{+}
$$

into the negative eigenspan, kernel, and positive eigenspan of $A_{b}$. The relation $\gamma A_{b}=-A_{b} \gamma$ implies that ker $A_{b}$ is preserved by $\gamma$ and that $\gamma$ maps $F_{b}^{+}$ unitarily onto $F_{b}^{-}$.

The kernel of $A_{b}$ is identified by the Hodge theorem with the twisted de Rham cohomology of the complex $\left(\Omega^{*}\left(\Sigma ;\left.E\right|_{\Sigma}\right), d_{b}\right)$; indeed the elements of ker $A_{b}$ are just the $d_{b}$-harmonic forms and so the composite

$$
\operatorname{ker} A_{b}=\operatorname{ker} d_{b} \cap \operatorname{ker} d_{b}^{*} \subset \operatorname{ker} d_{b} \rightarrow \frac{\operatorname{ker} d_{b}}{\text { image } d_{b}}
$$

is an isomorphism. The de Rham theorem then identifies the cohomology of $\left(\Omega^{*}\left(\Sigma ;\left.E\right|_{\Sigma}\right), d_{b}\right)$ and the (singular or cellular) cohomology $H^{*}\left(\Sigma ; \mathbb{C}_{\alpha}^{n}\right)$ with local coefficients given by the holonomy representation $\alpha$.

The triple $\left(\operatorname{ker} A_{b},\langle\rangle,, \gamma\right)$ gives ker $A_{b}$ the structure of a Hermitian symplectic space. In general a Hermitian symplectic space $(H,\langle\rangle,, \gamma)$ is a finite dimensional complex vector space $H$ with a positive definite Hermitian inner product $\langle\rangle:, H \times H \rightarrow \mathbb{C}$ and an isomorphism $\gamma: H \rightarrow H$ which is unitary, i.e. $\langle\gamma(x), \gamma(y)\rangle=\langle x, y\rangle$, satisfying $\gamma^{2}=-I$ such that the signature of $i \gamma$ is zero. The underlying symplectic structure is the pair $(H, \omega)$, where $\omega$ is the non-degenerate skew-Hermitian form

$$
\omega(x, y)=\langle x, \gamma(y)\rangle \text {. }
$$

The signature of $i \gamma$ on ker $A_{b} \cong H^{*}\left(\Sigma ; \mathbb{C}_{\alpha}^{n}\right)$ is zero. This is a consequence of the fact that $\left(\Sigma,\left.\alpha\right|_{\Sigma}\right)$ bounds $(X, \alpha)$, and is not true for a general pair $(\Sigma, \alpha)$. 
However, it is true in many important cases, for example if $\Sigma$ is a $4 \ell-2$ dimensional manifold and $\alpha: \pi_{1} \Sigma \rightarrow U(n)$ factors through $O(n)$.

In contrast to the Hermitian inner product $\langle$,$\rangle and the unitary map \gamma$ on ker $A_{b}$, the symplectic form $\omega$ does not depend on the Riemannian metric and in fact is given by the cup product:

$$
\omega\left(\beta_{1}, \beta_{2}\right)=i^{r} \int_{\Sigma} \beta_{1} \wedge \beta_{2}=i^{r}\left(\left[\beta_{1}\right] \cup\left[\beta_{2}\right]\right) \cap[\Sigma],
$$

where $i^{r}$ is a constant depending on the degrees of the $\beta_{i}$.

A subspace $W$ of a Hermitian symplectic space $(H, \omega)$ is called Lagrangian if $\omega$ vanishes on $W$ and $W$ is maximal with this property. This is equivalent to $\gamma(W)=W^{\perp}$, but being a Lagrangian subspace is a property of the underlying symplectic structure. Note that $\operatorname{dim} W=\frac{1}{2} \operatorname{dim} H$. Denote the Grassmannian of all Lagrangian subspaces of $H$ by $\mathscr{L}(H)$.

We summarize: The symplectic structure on $H^{*}\left(\Sigma ; \mathbb{C}_{\alpha}^{n}\right)$, and hence the Grassmannian $\mathscr{L}\left(H^{*}\left(\Sigma ; \mathbb{C}_{\alpha}^{n}\right)\right)$, depends only on the cohomology and cup product, and therefore is a homotopy invariant of $(\Sigma, \alpha)$. On the other hand, the Hermitian symplectic structure on $H^{*}\left(\Sigma ; \mathbb{C}_{\alpha}^{n}\right)$ depends on its identification with ker $A_{b}$ via the Hodge and de Rham theorems, since the inner product $\langle$, is the restriction of the $L^{2}$ inner product (which depends on the Riemannian metric on $\Sigma$ ) to $\operatorname{ker} A_{b}$.

The following lemma is well-known; it follows by a standard argument using Poincaré duality (cf. also [26, Cor. 8.4]).

Lemma 2.1 The image of the restriction

$$
H^{*}\left(X ; \mathbb{C}_{\alpha}^{n}\right) \rightarrow H^{*}\left(\Sigma ; \mathbb{C}_{\alpha}^{n}\right)
$$

is a Lagrangian subspace.

We will denote this subspace by $V_{X, \alpha}$, and, by slight abuse of notation, its preimage in $\operatorname{ker} A_{b}$ via the isomorphism ker $A_{b} \cong H^{*}\left(\Sigma ; \mathbb{C}_{\alpha}^{n}\right)$ will also be denoted by $V_{X, \alpha}$. We emphasize that the Lagrangian $V_{X, \alpha}$ is a homotopy invariant of $(X, \alpha)$. Moreover it gives a distinguished element in the Grassmannian $\mathscr{L}\left(H^{*}\left(\Sigma ; \mathbb{C}_{\alpha}^{n}\right)\right)$. Considered as a subspace of ker $A_{b}, V_{X, \alpha}$ coincides with the limiting values of extended $L^{2}$ solutions of $D_{B} \phi=0$ on $(\Sigma \times(-\infty, 0]) \cup X$ in the sense of [2].

Lagrangian subspaces of $H^{*}\left(\Sigma ; \mathbb{C}_{\alpha}^{n}\right)$ are used to produce elliptic self-adjoint Atiyah-Patodi-Singer (APS) boundary conditions for the odd signature operator $D_{B}$ as follows. Given a Lagrangian subspace $W \subset H^{*}\left(\Sigma ; \mathbb{C}_{\alpha}^{n}\right)$ we consider 
the orthogonal projection in $L^{2}\left(\Omega^{*}\left(\Sigma ;\left.E\right|_{\Sigma}\right)\right)$ onto $F_{b}^{+} \oplus W$. This orthogonal projection defines a well-posed boundary condition for $D_{B}$ (see e.g. [9]).

Restricting $D_{B}$ to the subspace of sections whose restriction to the boundary lies in the kernel of this projection makes $D_{B}$ a discrete self-adjoint operator which we denote by $D_{B, W}$. The following properties of this operator are the starting point of the investigations of this article and go back to Atiyah, Patodi, and Singer's fundamental articles [2, 3, 4]. In this context the following facts are explained in [26].

(1) The $\eta$ function of the operator $D_{B, W}$,

$$
\eta(s)=\sum_{\lambda \in \operatorname{Spec}\left(D_{B, W}\right) \backslash\{0\}} \operatorname{sign} \lambda|\lambda|^{-s},
$$

converges for $\operatorname{Re}(s)>>0$ and has a meromorphic continuation to the entire complex plane with no pole at $s=0$. Denote its value at $s=0$ by

$$
\eta\left(D_{B, W}, X\right):=\eta(0) .
$$

(2) The kernel of $D_{B, W}$ fits into an exact sequence

$$
\begin{aligned}
0 \rightarrow\left(\text { image } H^{*}\left(X, \Sigma ; \mathbb{C}_{\alpha}^{n}\right)\right. & \left.\rightarrow H^{*}\left(X ; \mathbb{C}_{\alpha}^{n}\right)\right) \rightarrow \ldots \\
\ldots & \rightarrow \operatorname{ker} D_{B, W} \rightarrow W \cap \gamma\left(V_{X, \alpha}\right) \rightarrow 0 .
\end{aligned}
$$

In particular, taking $W=V_{X, \alpha}$ we see

$$
\text { ker } D_{B, V_{X, \alpha}}=\text { image } H^{*}\left(X, \Sigma ; \mathbb{C}_{\alpha}^{n}\right) \rightarrow H^{*}\left(X ; \mathbb{C}_{\alpha}^{n}\right) \text {. }
$$

We next recall the definition of the $\rho$-invariant for manifolds with boundary from [26]. Let $\Theta$ denote the trivial connection in the product bundle $\mathbb{C}^{n} \times X$ in the form $\Theta=\pi^{*}(\theta)$ in the collar of $\partial X$, and $\tau: \pi_{1} X \rightarrow U(n)$ the trivial representation. Then define

$$
\rho(X, \alpha, g)=\eta\left(D_{B, V_{X, \alpha}}, X\right)-\eta\left(D_{\Theta, V_{X, \tau}}, X\right) .
$$

It is shown in $[26$, Sec. 8$]$ that $\rho(X, \alpha, g)$ depends only on the smooth structure on $X$, the conjugacy class of $\alpha: \pi_{1} X \rightarrow U(n)$, and the Riemannian metric $g$ on $\Sigma=\partial X$. In particular, it is independent of the choice of flat connection $B$ with holonomy conjugate to $\alpha$ and also independent of the Riemannian metric $\tilde{g}$ on $X$ extending $g$.

When $\partial X$ is empty, then the diffeomorphism invariance of $\rho(X, \alpha)$ was established by Atiyah, Patodi, and Singer in [3] and follows straightforwardly from their index theorem. The cut-and-paste formulae

$$
\eta\left(D_{B}, M\right)=\eta\left(D_{B, V_{X, \alpha}}, X\right)+\eta\left(D_{B, V_{Y, \alpha}}, Y\right)+m\left(V_{X, \alpha}, V_{Y, \alpha}\right)_{(b, g)}
$$


and

$$
\rho(M, \alpha)=\rho(X, \alpha, g)+\rho(Y, \alpha, g)+m\left(V_{X, \alpha}, V_{Y, \alpha}\right)_{(b, g)}-m\left(V_{X, \tau}, V_{Y, \tau}\right)_{(\theta, g)}
$$

when $M=Y \cup_{\Sigma} X$ were proven in [26, Sec. 8] and are the basis for our investigations in the present article.

In Equations (2.5) and (2.6) the correction term $m(V, W)_{(b, g)}$ is a real valued invariant of pairs of Lagrangians in $H^{*}\left(\Sigma ; \mathbb{C}_{\alpha}^{n}\right)$; it depends on the identification of $H^{*}\left(\Sigma ; \mathbb{C}_{\alpha}^{n}\right)$ with the kernel of $A_{b}$ and hence may a priori (and a posteriori as well, see Section 6) depend on the Riemannian metric $g$ on $\Sigma$. It is defined as follows.

Let $\operatorname{ker} A_{b}^{+}$denote the $+i$-eigenspace of $\gamma$ acting on $\operatorname{ker} A_{b}$ and let $\operatorname{ker} A_{b}^{-}$ denote the $-i$-eigenspace. Then every Lagrangian subspace $W$ of ker $A_{b} \cong$ $H^{*}\left(\Sigma ; \mathbb{C}_{\alpha}^{n}\right)$ can be written uniquely as a graph

$$
W=\left\{x+\phi(W)(x) \mid x \in \operatorname{ker} A_{b}^{+}\right\},
$$

where $\phi(W): \operatorname{ker} A_{b}^{+} \rightarrow \operatorname{ker} A_{b}^{-}$is a unitary isomorphism. The map $W \mapsto$ $\phi(W)$ determines a diffeomorphism between the space $\mathscr{L}\left(\operatorname{ker} A_{b}\right)$ of Lagrangians in $\operatorname{ker} A_{b}$ to the space of unitary operators $U\left(\operatorname{ker} A_{b}^{+}, \operatorname{ker} A_{b}^{-}\right)$. We take the branch $\log \left(r e^{i t}\right)=\ln r+i t, r>0,-\pi<t \leq \pi$ and use it to define $\operatorname{tr} \log : U\left(\operatorname{ker} A_{b}^{+}\right) \rightarrow i \mathbb{R}$ via $\operatorname{tr} \log (U)=\sum \log \left(\lambda_{i}\right), \lambda_{i} \in \operatorname{Spec} U$. Then define

$$
\begin{aligned}
m(V, W)_{(b, g)} & =-\frac{1}{\pi i} \operatorname{tr} \log \left(-\phi(V) \phi(W)^{*}\right)+\operatorname{dim}(V \cap W) \\
& =-\frac{1}{\pi i} \sum_{\substack{\lambda \in \operatorname{Spec}\left(-\phi(V) \phi(W)^{*}\right) \\
\lambda \neq-1}} \log \lambda .
\end{aligned}
$$

We will abbreviate this to $m(V, W)$ when $(b, g)$ is clear from context. Since $-\phi(V) \phi(W)^{*}$ is unitary, its eigenvalues are unit complex numbers, and hence $m(V, W)$ is a real number. The term $\operatorname{dim}(V \cap W)$ is added to match conventions and to simplify formulas; notice that its effect is to remove the contribution of the -1 eigenspace of $-\phi(V) \phi(W)^{*}$ to $\operatorname{tr} \log \left(-\phi(V) \phi(W)^{*}\right)$. Thus $m$ is not in general a continuous function of $V$ and $W$. The function $m$ has been investigated before, the notation is taken from [10].

\section{Cutting and pasting formulas with arbitrary boundary conditions}

The $\eta$-invariants appearing in the definition of $\rho$ of Equation (2.4) are taken with respect to the boundary conditions $V_{X, \alpha} \subset H^{*}\left(\Sigma ; \mathbb{C}_{\alpha}^{n}\right)$ and $V_{X, \tau} \subset$ 
$H^{*}\left(\Sigma ; \mathbb{C}^{n}\right)$. More precisely, the Lagrangian $V_{X, \alpha} \subset H^{*}\left(\Sigma ; \mathbb{C}_{\alpha}^{n}\right)$ determines a subspace (still denoted $V_{X, \alpha}$ ) of ker $A_{b}$, and this in turn determines the orthogonal projection to $F_{b}^{+} \oplus V_{X, \alpha}$, (recall that $F_{b}^{+}$is shorthand for the positive eigenspan of $A_{b}$ ). A similar comment applies to $V_{X, \tau}$. Since these Lagrangians are canonically determined by the homotopy type of the pair $(X, \alpha)$ and the Riemannian metric on $\Sigma$, they present a natural choice for the boundary conditions. Nevertheless it is useful to use other Lagrangians in $H^{*}\left(\Sigma ; \mathbb{C}_{\alpha}^{n}\right)$ to define boundary conditions. One important reason is that the $V_{X, \alpha}$ do not vary continuously in families, even if $\operatorname{ker} A_{b}$ does.

Definition 3.1 Let $X$ have boundary $\Sigma$ and let $\alpha: \pi_{1} X \rightarrow U(n)$ be a representation. Given Lagrangian subspaces $W_{\alpha} \subset H^{*}\left(\Sigma ; \mathbb{C}_{\alpha}^{n}\right)$ and $W_{\tau} \subset$ $H^{*}\left(\Sigma ; \mathbb{C}^{n}\right)$, define $\rho\left(X, \alpha, g, W_{\alpha}, W_{\tau}\right)$ by

$$
\rho\left(X, \alpha, g, W_{\alpha}, W_{\tau}\right):=\eta\left(D_{B, W_{\alpha}}, X\right)-\eta\left(D_{\Theta, W_{\tau}}, X\right) .
$$

Thus $\rho(X, \alpha, g)$ is shorthand for $\rho\left(X, \alpha, g, V_{X, \alpha}, V_{X, \tau}\right)$.

We next recall the definition of $\tilde{\sigma}$ from [26, Sec. 8]. Given Lagrangian subspaces $U, V, W$ of a Hermitian symplectic space, define

$$
\tilde{\sigma}(U, V, W):=m(U, V)+m(V, W)+m(W, U) .
$$

Then $\tilde{\sigma}$ is integer-valued, depends only on the symplectic form $\omega$, and coincides with Wall's correction term for the non-additivity of the signature [36] as well as the Maslov triple index of [11].

The following theorem gives a complete formulation of the dependence of the $\eta$ - and $\rho$-invariants for a manifold with boundary on the choice of Lagrangians used for APS boundary conditions.

Theorem 3.2 Suppose that $M=Y \cup_{\Sigma} X, \alpha: \pi_{1}(M) \rightarrow U(n)$ is a representation, $W_{\alpha}^{X}, W_{\alpha}^{Y} \subset H^{*}\left(\Sigma ; \mathbb{C}_{\alpha}^{n}\right)$ and $W_{\tau}^{X}, W_{\tau}^{Y} \subset H^{*}\left(\Sigma ; \mathbb{C}^{n}\right)$ are Lagrangian subspaces, and let $B$ be a flat connection on $M$ with holonomy $\alpha$ in cylindrical form near $\Sigma$. Orientation dependent quantities like $\gamma$ etc. are taken with respect to $X$ according to the convention explained on page 629 .

Then:

(1) $\eta\left(D_{B, W_{\alpha}^{X}}, X\right)-\eta\left(D_{B, V_{X, \alpha}}, X\right)=m\left(\gamma\left(V_{X, \alpha}\right), W_{\alpha}^{X}\right)$.

(2) $\rho\left(X, \alpha, g, W_{\alpha}^{X}, W_{\tau}^{X}\right)$ depends only on the diffeomorphism type of $X$, the representation $\alpha$, the Lagrangian subspaces $W_{\alpha}^{X}, W_{\tau}^{X}$ and the Riemannian metric $g$ on $\Sigma=\partial X$. 
(3) The difference $\eta\left(D_{B}, M\right)-\eta\left(D_{B, W_{\alpha}^{X}}, X\right)-\eta\left(D_{B, W_{\alpha}^{Y}}, Y\right)-m\left(W_{\alpha}^{X}, W_{\alpha}^{Y}\right)$ is an integer. In fact it equals

$$
\tilde{\sigma}\left(V_{X, \alpha}, V_{Y, \alpha}, \gamma\left(W_{\alpha}^{Y}\right)\right)-\tilde{\sigma}\left(\gamma\left(V_{X, \alpha}\right), W_{\alpha}^{X}, W_{\alpha}^{Y}\right) .
$$

(4) $\eta\left(D_{B}, M\right)=\eta\left(D_{B, V_{X, \alpha}}, X\right)+\eta\left(D_{B, \gamma\left(V_{X, \alpha}\right)}, Y\right)$ and so

$$
\rho(M, \alpha)=\rho\left(X, \alpha, V_{X, \alpha}, V_{X, \tau}\right)+\rho\left(Y, \alpha, \gamma\left(V_{X, \alpha}\right), \gamma\left(V_{X, \tau}\right)\right) .
$$

Proof We use the results of [26]. Recall the notation $\tilde{\eta}(D)=\frac{1}{2}(\eta(D)+$ dim ker $D)$. For the proof of (1) we omit the sub- and superscripts of $W$ :

By [26, Theorem 4.4] we have

$$
\begin{aligned}
& \tilde{\eta}\left(D_{B, W}, X\right)-\tilde{\eta}\left(D_{B, V_{X, \alpha}}, X\right) \\
& \quad=\frac{1}{2 \pi i}\left(\operatorname{tr} \log \left(\Phi\left(P^{+}(W)\right) \Phi\left(P_{X}\right)^{*}\right)-\operatorname{tr} \log \left(\Phi\left(P^{+}\left(V_{X, \alpha}\right)\right) \Phi\left(P_{X}\right)^{*}\right)\right) .
\end{aligned}
$$

Here, $P^{+}(W)$ denotes the orthogonal projection onto $W \oplus F_{b}^{+}, P_{X}$ denotes the Calderòn projector for $D_{B}$ acting on $X$, and $\Phi$ is the infinite-dimensional version of $\phi$ : it denotes the diffeomorphism from the (infinite-dimensional) Lagrangian Grassmannian onto $\mathscr{U}(\operatorname{ker}(\gamma-i), \operatorname{ker}(\gamma+i))$ (cf. [26, Sec. 2]). Using [26, Lemma 6.9] we identify the right side of (3.1) with

$$
\tau_{\mu}\left(P^{+}\left(V_{X, \alpha}\right), P^{+}(W), P_{X}\right)-\frac{1}{2 \pi i} \operatorname{tr} \log \left(\Phi\left(P^{+}\left(V_{X, \alpha}\right)\right) \Phi\left(P^{+}(W)\right)^{*}\right),
$$

where $\tau_{\mu}$ is the Maslov triple index defined in [26, Sec. 6].

In view of $\left[26\right.$, Lemma 8.10] the quantity $\tau_{\mu}\left(P^{+}\left(V_{X, \alpha}\right), P^{+}(W), P_{X}\right)$ is invariant under adiabatic stretching and equals

$$
\tau_{\mu}\left(V_{X, \alpha}, W, V_{X, \alpha}\right)=\operatorname{dim}\left(V_{X, \alpha} \cap \gamma(W)\right),
$$

where the last equality follows from [26, Prop. 6.11].

As in the proof of [26, Theorem 8.12] one calculates

$$
\operatorname{tr} \log \left(\Phi\left(P^{+}\left(V_{X, \alpha}\right)\right) \Phi\left(P^{+}(W)^{*}\right)\right)=\operatorname{tr} \log \left(\phi\left(V_{X, \alpha}\right) \phi(W)^{*}\right) .
$$

The identity $\gamma^{2}=-I$ shows that $\operatorname{dim}\left(V_{X, \alpha} \cap \gamma(W)\right)=\operatorname{dim}\left(\gamma\left(V_{X, \alpha}\right) \cap W\right)$ and clearly $\phi(\gamma(W))=-\phi(W)$. These facts together with the definition of $m(V, W)$ and Equation (3.4) imply

$$
\begin{aligned}
\tilde{\eta}\left(D_{B, W}, X\right) & -\tilde{\eta}\left(D_{B, V_{X, \alpha}}, X\right) \\
& =\operatorname{dim}\left(\gamma\left(V_{X, \alpha}\right) \cap W\right)-\frac{1}{2 \pi i} \operatorname{tr} \log \left(\phi\left(V_{X, \alpha}\right) \phi(W)^{*}\right) \\
& =\frac{1}{2}\left(m\left(\gamma\left(V_{X, \alpha}\right), W\right)+\operatorname{dim}\left(\gamma\left(V_{X, \alpha}\right) \cap W\right)\right) .
\end{aligned}
$$


Using the definition $\tilde{\eta}(D)=\frac{1}{2}(\eta(D)+\operatorname{dim} \operatorname{ker} D)$ we see that $\eta\left(D_{B, W}, X\right)-$ $\eta\left(D_{B, V_{X, \alpha}}, X\right)-m\left(\gamma\left(V_{X, \alpha}\right), W\right)$ equals

$$
-\operatorname{dim} \operatorname{ker} D_{B, W}+\operatorname{dim} \operatorname{ker} D_{B, V_{X, \alpha}}+\operatorname{dim}\left(\gamma\left(V_{X, \alpha}\right) \cap W\right) .
$$

But (3.6) vanishes, as one sees by using the exact sequence (2.3). This proves the first assertion of Theorem 3.2.

The second assertion follows from the first part and [26, Lemma 8.15].

Using (2.5) and the first assertion one sees that

$$
\eta\left(D_{B}, M\right)-\eta\left(D_{B, W_{\alpha}^{X}}, X\right)-\eta\left(D_{B, W_{\alpha}^{Y}}, Y\right)-m\left(W_{\alpha}^{X}, W_{\alpha}^{Y}\right)
$$

equals

$$
m\left(V_{X, \alpha}, V_{Y, \alpha}\right)-m\left(\gamma\left(V_{X, \alpha}\right), W_{\alpha}^{X}\right)+m\left(\gamma\left(V_{Y, \alpha}\right), W_{\alpha}^{Y}\right)-m\left(W_{\alpha}^{X}, W_{\alpha}^{Y}\right) .
$$

(There is one subtlety: the sign change of the term $m\left(\gamma\left(V_{Y, \alpha}\right), W_{\alpha}^{Y}\right)$ occurs because viewed from the " $Y$ " side, the Hermitian symplectic structure changes sign.)

Using the identities $m(V, W)=-m(W, V)$ and $\phi(\gamma(W))=-\phi(W)$, so that $m(\gamma(V), \gamma(W))=m(V, W)$, we can rewrite $(3.7)$ as

$$
-m\left(\gamma\left(V_{X, \alpha}\right), W_{\alpha}^{X}\right)-m\left(W_{\alpha}^{X}, W_{\alpha}^{Y}\right)+m\left(V_{X, \alpha}, V_{Y, \alpha}\right)+m\left(V_{Y, \alpha}, \gamma\left(W_{\alpha}^{Y}\right)\right),
$$

which equals

$$
\begin{aligned}
& \tilde{\sigma}\left(V_{X, \alpha}, V_{Y, \alpha}, \gamma\left(W_{\alpha}^{Y}\right)\right)-m\left(\gamma\left(W_{\alpha}^{Y}\right), V_{X, \alpha}\right) \\
& -\tilde{\sigma}\left(\gamma\left(V_{X, \alpha}\right), W_{\alpha}^{X}, W_{\alpha}^{Y}\right)+m\left(W_{\alpha}^{Y}, \gamma\left(V_{X, \alpha}\right)\right) \\
= & \tilde{\sigma}\left(V_{X, \alpha}, V_{Y, \alpha}, \gamma\left(W_{\alpha}^{Y}\right)\right)-\tilde{\sigma}\left(\gamma\left(V_{X, \alpha}\right), W_{\alpha}^{X}, W_{\alpha}^{Y}\right)
\end{aligned}
$$

as desired. This proves the third assertion.

The last statement follows straightforwardly from the previous or, alternatively, can be immediately recovered from [26, Theorem 8.8].

\section{Lagrangians induced by bordisms}

Theorem 3.2 gives splitting formulas for the $\eta$ and $\rho$-invariants of $D_{B}$ in the situation when a manifold $M$ is decomposed into two pieces $X$ and $Y$ along a hypersurface $\Sigma$. To develop this into a useful cut-and-paste machinery for the $\rho$-invariant requires keeping track of the Lagrangian subspaces $V_{X, \alpha}=$ image $H^{*}\left(X ; \mathbb{C}_{\alpha}^{n}\right) \rightarrow H^{*}\left(\Sigma ; \mathbb{C}_{\alpha}^{n}\right)$ and their generalizations. It is clearest to give 
an exposition based on the effect of a bordism on Lagrangian subspaces and we do this next.

Let $X$ be a Riemannian manifold with boundary $-\Sigma_{0} \amalg \Sigma_{1}$ (we allow $\Sigma_{0}$ or $\Sigma_{1}$ empty). Let $\alpha: \pi_{1} X \rightarrow U(n)$ be a representation. Fix a flat connection $B$ on $X$ with holonomy $\alpha$ in cylindrical form near $\Sigma_{0}$ and $\Sigma_{1}$. The tangential operator $A_{b}$ of $D_{B}$ acting on $X$ decomposes as a direct sum $A_{b}=A_{b, 0} \oplus A_{b, 1}$ since $L^{2}(\partial X)=L^{2}\left(\Sigma_{0}\right) \oplus L^{2}\left(\Sigma_{1}\right)$. In particular

$$
\operatorname{ker} A_{b}=\operatorname{ker} A_{b, 0} \oplus A_{b, 1} \cong H^{*}\left(\Sigma_{0} ; \mathbb{C}_{\alpha}^{n}\right) \oplus H^{*}\left(\Sigma_{1} ; \mathbb{C}_{\alpha}^{n}\right) .
$$

We view $X$ as a bordism from $\Sigma_{0}$ to $\Sigma_{1}$.

We explained in the previous section that $\operatorname{ker} A_{b} \cong H^{*}\left(\partial X ; \mathbb{C}_{\alpha}^{n}\right)$ is a Hermitian symplectic space. At this point we add the hypothesis that both $\operatorname{ker} A_{b, 0} \cong$ $H^{*}\left(\Sigma_{0} ; \mathbb{C}_{\alpha}^{n}\right)$ and ker $A_{b, 1} \cong H^{*}\left(\Sigma_{1} ; \mathbb{C}_{\alpha}^{n}\right)$ be Hermitian symplectic spaces. This is not automatic, but follows for example if there exists a manifold $Y$ with boundary $\Sigma_{0}$ over which $\left.\alpha\right|_{\Sigma_{0}}: \pi_{1} \Sigma_{0} \rightarrow U(n)$ extends. It is in this context that we will usually work.

We use $X$ to define a function $L_{X, \alpha}$ from the set of subspaces of $H^{*}\left(\Sigma_{0} ; \mathbb{C}_{\alpha}^{n}\right)$ to the set of subspaces of $H^{*}\left(\Sigma_{1} ; \mathbb{C}_{\alpha}^{n}\right)$ by

$$
L_{X, \alpha}(W)=P_{1}\left(V_{X, \alpha} \cap\left(W \oplus H^{*}\left(\Sigma_{1} ; \mathbb{C}_{\alpha}^{n}\right)\right)\right),
$$

where $P_{1}: H^{*}\left(\partial X ; \mathbb{C}_{\alpha}^{n}\right) \rightarrow H^{*}\left(\Sigma_{1} ; \mathbb{C}_{\alpha}^{n}\right)$ denotes the projection onto the second factor:

$$
P_{1}: H^{*}\left(\partial X ; \mathbb{C}_{\alpha}^{n}\right)=H^{*}\left(\Sigma_{0} ; \mathbb{C}_{\alpha}^{n}\right) \oplus H^{*}\left(\Sigma_{1} ; \mathbb{C}_{\alpha}^{n}\right) \rightarrow H^{*}\left(\Sigma_{1} ; \mathbb{C}_{\alpha}^{n}\right) .
$$

In the following theorem, let $Y$ be a Riemannian manifold with boundary $\Sigma_{0}$ with a product metric $g_{0}+d u^{2}$ near the collar. Write

$$
Z=Y \cup_{\Sigma_{0}} X
$$

and assume that $\alpha$ extends over $Z$. Let $\gamma_{0}$ be the restriction of $\gamma$ to ker $A_{b, 0}$. Notice that $\gamma_{0}\left(V_{Y, \alpha}\right) \oplus L_{X, \alpha}\left(V_{Y, \alpha}\right)$ is a Lagrangian subspace of ker $A_{b}$.

Theorem 4.1 The function of Equation (4.1) takes Lagrangian subspaces to Lagrangian subspaces, i.e. it induces a function

$$
L_{X, \alpha}: \mathscr{L}\left(H^{*}\left(\Sigma_{0} ; \mathbb{C}_{\alpha}^{n}\right)\right) \rightarrow \mathscr{L}\left(H^{*}\left(\Sigma_{1} ; \mathbb{C}_{\alpha}^{n}\right)\right) .
$$

This function has the properties: 
(1) If $Y, Z=Y \cup_{\Sigma_{0}} X$ are as above then

$$
V_{Z, \alpha}=L_{X, \alpha}\left(V_{Y, \alpha}\right) .
$$

In short, the bordism propagates the distinguished Lagrangian. Moreover

$$
\eta\left(D_{B, V_{Z, \alpha}}, Z\right)=\eta\left(D_{B, V_{Y, \alpha}}, Y\right)+\eta\left(D_{B, \gamma_{0}}\left(V_{Y, \alpha}\right) \oplus L_{X, \alpha}\left(V_{Y, \alpha}\right), X\right)
$$

and hence $\rho\left(Z, \alpha, g_{1}\right)$ equals

$\rho\left(Y, \alpha, g_{0}\right)+\rho\left(X, \alpha, g_{0} \amalg g_{1}, \gamma_{0}\left(V_{Y, \alpha}\right) \oplus L_{X, \alpha}\left(V_{Y, \alpha}\right), \gamma_{0}\left(V_{Y, \tau}\right) \oplus L_{X, \tau}\left(V_{Y, \tau}\right)\right)$.

where $g_{i}$ is a metric on $\Sigma_{i}$.

(2) If $X_{1}$ is a bordism from $\Sigma_{0}$ to $\Sigma_{1}$ and $X_{2}$ is a bordism from $\Sigma_{1}$ to $\Sigma_{2}$ and $\alpha: \pi_{1}\left(X_{1} \cup_{\Sigma_{1}} X_{2}\right) \rightarrow U(n)$ then

$$
L_{X_{1} \cup_{\Sigma_{1}} X_{2}, \alpha}=L_{X_{2}, \alpha} \circ L_{X_{1}, \alpha} .
$$

Proof The map of (4.1) is just the map taking $V_{X, \alpha}$ to its symplectic reduction with respect to the subspace $W \oplus H^{*}\left(\Sigma_{1} ; \mathbb{C}_{\alpha}^{n}\right) \subset H^{*}\left(\Sigma_{0} ; \mathbb{C}_{\alpha}^{n}\right) \oplus H^{*}\left(\Sigma_{1} ; \mathbb{C}_{\alpha}^{n}\right)$ (cf. [26, Sec. 6.3]). Symplectic reduction takes Lagrangians to Lagrangians.

To prove the the first part of (1) consider a $\xi \in V_{Y \cup_{\Sigma_{0}} X, \alpha}$. Then there is a $w \in H^{*}\left(Y \cup_{\Sigma_{0}} X ; \mathbb{C}_{\alpha}^{n}\right)$ with $i_{\Sigma_{1}}^{*} w=\xi$. We put $\xi_{0}:=-i_{\Sigma_{0}}^{*} w$. Since certainly $w_{\mid X} \in H^{*}\left(X ; \mathbb{C}_{\alpha}^{n}\right)$ we infer $\xi_{0} \oplus \xi=i_{\partial X}^{*} w \in V_{X, \alpha}$. Thus $\xi=P_{1}\left(\xi_{0} \oplus \xi\right) \in$ $P_{1}\left(V_{X, \alpha} \cap\left(V_{Y, \alpha} \oplus H^{*}\left(\Sigma_{1} ; \mathbb{C}_{\alpha}^{n}\right)\right)\right)$.

Conversely, let $\xi \in P_{1}\left(V_{X, \alpha} \cap\left(V_{Y, \alpha} \oplus H^{*}\left(\Sigma_{1} ; \mathbb{C}_{\alpha}^{n}\right)\right)\right)$ be given. Then there is $\xi_{0} \in V_{Y, \alpha}$ such that $\xi_{0} \oplus \xi \in V_{X, \alpha}$. Thus we may choose $w_{X} \in H^{*}\left(X ; \mathbb{C}_{\alpha}^{n}\right)$ with $i_{\partial X}^{*} w_{X}=\xi_{0} \oplus \xi$ and $w_{Y} \in H^{*}\left(Y ; \mathbb{C}_{\alpha}^{n}\right)$ with $i_{\Sigma_{0}}^{*} w_{Y}=\xi_{0}$.

From the Mayer-Vietoris sequence of $Y \cup_{\Sigma_{0}} X$ we obtain an $w \in H^{*}\left(Y \cup_{\Sigma_{0}}\right.$ $\left.X ; \mathbb{C}_{\alpha}^{n}\right)$ with $w_{\mid Y}=w_{Y}$ and $w_{\mid X}=w_{X}$. Then $\xi=i_{\Sigma_{1}}^{*} w_{X}=i_{\Sigma_{1}}^{*} w \in V_{Y \cup_{\Sigma_{0}} X, \alpha}$ and we reach the conclusion.

Consider now the second part of (1). We have explained in [26, Sec. 7] that the gluing formula for $\eta$-invariants remain true if one glues (a finite union of) components of the boundary and fixes a boundary condition at the remaining components. The result now follows from $V_{Z, \alpha}=L_{X, \alpha}\left(V_{Y, \alpha}\right)$ and Theorem 3.2.

The proof of (2) proceeds along the same lines as the proof of the first part of (1). Consider $W \subset H^{*}\left(\Sigma_{0} ; \mathbb{C}_{\alpha}^{n}\right)$ and a $\xi \in L_{X_{1} \cup_{\Sigma_{1}} X_{2}, \alpha}(W)$. Then there is a $w \in H^{*}\left(X_{1} \cup \Sigma_{\Sigma_{1}} X_{2} ; \mathbb{C}_{\alpha}^{n}\right)$ with $i_{\Sigma_{2}}^{*} w=\xi$ and $\xi_{0}:=-i_{\Sigma_{0}}^{*} w \in W$. Put $\xi_{1}:=i_{\Sigma_{1}}^{*} w$. Then it is immediate that $\xi_{0} \oplus \xi_{1} \in V_{X_{1}, \alpha} \cap\left(W \oplus H^{*}\left(\Sigma_{1} ; \mathbb{C}_{\alpha}^{n}\right)\right)$ and $-\xi_{1} \oplus \xi \in V_{X_{2}, \alpha} \cap\left(L_{X_{1}, \alpha}(W) \oplus H^{*}\left(\Sigma_{2} ; \mathbb{C}_{\alpha}^{n}\right)\right)$. This proves $\xi \in L_{X_{2}, \alpha} \circ L_{X_{1}, \alpha}(W)$. 
Conversely, let $\xi \in L_{X_{2}, \alpha} \circ L_{X_{1}, \alpha}(W)$ be given. Then there exists a $\xi_{1} \in$ $H^{*}\left(\Sigma_{1} ; \mathbb{C}_{\alpha}^{n}\right)$ such that $-\xi_{1} \oplus \xi \in V_{X_{2}, \alpha} \cap\left(L_{X_{1}, \alpha}(W) \oplus H^{*}\left(\Sigma_{2} ; \mathbb{C}_{\alpha}^{n}\right)\right)$ and a $\xi_{0} \in$ $H^{*}\left(\Sigma_{0} ; \mathbb{C}_{\alpha}^{n}\right)$ such that $-\xi_{0} \oplus \xi_{1} \in V_{X_{1}, \alpha} \cap\left(W \oplus H^{*}\left(\Sigma_{1}, \mathbb{C}_{\alpha}^{n}\right)\right)$.

A Mayer-Vietoris argument as in the proof of the first part of (1) shows the existence of a $w \in H^{*}\left(X_{1} \cup_{\Sigma_{1}} X_{2} ; \mathbb{C}_{\alpha}^{n}\right)$ such that $i_{\Sigma_{2}}^{*} w=\xi$ and $i_{\Sigma_{0}}^{*} w=-\xi_{0}$. This proves $-\xi_{0} \oplus \xi \in V_{X_{1} \cup_{\Sigma_{1}} X_{2}, \alpha} \cap\left(W \oplus H^{*}\left(\Sigma_{2} ; \mathbb{C}_{\alpha}^{n}\right)\right)$, and hence $L_{X_{2}, \alpha} \circ$ $L_{X_{1}, \alpha}(W) \subset L_{X_{1} \cup_{\Sigma_{1}} X_{2}, \alpha}(W)$.

Theorem 4.1 easily extends to the situation

$$
Z=Y \cup_{\Sigma_{0}} X_{1} \cup_{\Sigma_{1}} \cdots \cup_{\Sigma_{n}} X_{n+1}
$$

This gives a useful strategy for computing $\rho$-invariants by decomposing a closed manifold into a sequence of bordisms, e.g. by cutting along level sets of a Morse function.

We use Theorem 4.1 and the definitions to write down a formula which expresses the dependence of $\rho(Y, \alpha, g)$ on the metric $g$ on $\partial Y$.

Corollary 4.2 Let $Y$ be a compact manifold with boundary $\Sigma$. Let $\alpha: \pi_{1} Y \rightarrow$ $U(n)$ be a representation. Suppose that $g_{0}, g_{1}$ are two Riemannian metrics on $\Sigma$. Choose a path of metrics from $g_{0}$ to $g_{1}$ and view this path as a metric on $\Sigma \times[0,1]$.

Then

$$
\begin{aligned}
& \rho\left(Y, \alpha, g_{1}\right)-\rho\left(Y, \alpha, g_{0}\right) \\
& =\eta\left(D_{B, \gamma_{0}\left(V_{Y, \alpha}\right) \oplus V_{Y, \alpha}}, \Sigma \times[0,1]\right)-\eta\left(D_{\left.\Theta, \gamma_{0}\left(V_{Y, \tau}\right) \oplus V_{Y, \tau}, \Sigma \times[0,1]\right)}\right. \\
& =\rho\left(\Sigma \times[0,1], g_{0} \amalg g_{1}, \gamma_{0}\left(V_{Y, \alpha}\right) \oplus V_{Y, \alpha}, \gamma_{0}\left(V_{Y, \tau}\right) \oplus V_{Y, \tau}\right) \text {. }
\end{aligned}
$$

Here, $\Sigma$ is oriented such that a collar of the boundary takes the form $\Sigma \times(-\epsilon, 0]$.

Proof Apply Theorem 4.1 with $X=\Sigma \times[0,1]$ and note that for the cylinder $X=\Sigma \times[0,1]$ the map $L_{X, \alpha}$ is the identity.

In Section 6 we will use Corollary 4.2 to give examples that show that $\rho(Y, \alpha, g)$ depends in general on the choice of $g$, in contrast with the $\rho$-invariant for closed manifolds. 


\section{Diffeomorphism properties}

We next tie topology to the issue of the dependence of the $\rho$-invariant on the Riemannian metric on the boundary by exploiting the isometry invariance of spectral invariants.

Let $X$ be a manifold with boundary $\Sigma$. Suppose we are given a diffeomorphism $f: \Sigma \rightarrow \Sigma$ which extends to a diffeomorphism $F: X \rightarrow X$. We may assume that $F$ preserves a collar of the boundary. Then $F$ can be used to pull back flat connections and metrics. Moreover, the diagram

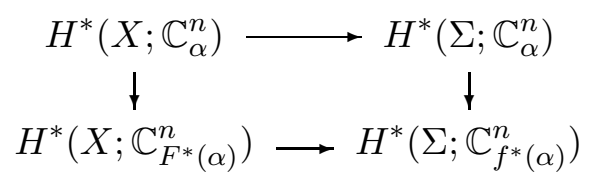

shows that $f^{*}\left(V_{X, \alpha}\right)=V_{X, F^{*}(\alpha)}$. Pulling back a metric on $X$ via $F$ gives an isometry which induces a unitary transformation on $L^{2}\left(\Omega_{X}^{e v}(E)\right)$ taking $D_{B}$ to $D_{\left.F^{*}(B)\right)}$ and taking $F_{b}^{+} \oplus V_{X, \alpha}$ to $F_{b}^{+} \oplus V_{X, F^{*}(\alpha)}$. Hence the spectra of the two operators are the same. Therefore

$$
\eta\left(D_{B, V_{X, \alpha}}, X\right)=\eta\left(D_{F^{*}(B), V_{X, F^{*}(\alpha)}}, X\right) .
$$

Applying this formula first with $B$ a flat connection with holonomy $\alpha$ and then with $B$ the trivial connection (and using the definition (2.4)) we immediately conclude the following.

Theorem 5.1 Let $X$ be a manifold with boundary $\Sigma$ and $\alpha: \pi_{1} X \rightarrow U(n)$ a representation. Let $g$ be a Riemannian metric on $\Sigma$. Suppose that $F: X \rightarrow X$ is a diffeomorphism. Let $f: \Sigma \rightarrow \Sigma$ denote its restriction to $\Sigma$. Then

$$
\rho(X, \alpha, g)=\rho\left(X, F^{*}(\alpha), f^{*}(g)\right) .
$$

As an example, recall that two diffeomorphisms $f_{0}, f_{1}: \Sigma \rightarrow \Sigma$ are pseudoisotopic if there exists a diffeomorphism $F: \Sigma \times[0,1] \rightarrow \Sigma \times[0,1]$ which restricts to $f_{0}$ and $f_{1}$ on the boundary. In particular an isotopy is a level-preserving pseudo-isotopy.

Corollary 5.2 The $\rho$-invariant for a manifold with boundary depends on the Riemannian metric on the boundary only up to its pseudo-isotopy class. Precisely, if $f_{0}, f_{1}: \partial X \rightarrow \partial X$ are pseudo-isotopic diffeomorphisms, then

$$
\rho\left(X, \alpha, f_{0}^{*}(g)\right)=\rho\left(X, \alpha, f_{1}^{*}(g)\right) .
$$


Proof We may assume $f_{0}$ is the identity by replacing $f_{1}$ by $f_{0}^{-1} \circ f_{1}$ and $g$ by $f_{0}^{*} g$. If $f_{0}$ is the identity then using a collar we see that $f_{1}$ extends to a diffeomorphism $F: X \rightarrow X$ which induces the identity on $\pi_{1} X$. Thus $F^{*}(\alpha)=\alpha$ and the claim follows from Theorem 5.1.

As another application, suppose that $F: X \rightarrow X$ is a diffeomorphism whose restriction to the boundary is the identity (or pseudo-isotopic to the identity). Then for any metric $g$ on $\Sigma$

$$
\rho(X, \alpha, g)=\rho\left(X, F^{*}(\alpha), g\right) .
$$

These facts can be summarized as follows. Let $\mathscr{M}_{\Sigma}$ denote the space of Riemannian metrics on $\Sigma$, and $\mathscr{F}_{X}$ the space of flat connections on $E \rightarrow X$. If $\partial X=\Sigma$, let $\mathscr{D}_{X}$ denote the diffeomorphism group of $X$ and let $\mathscr{D}_{X}^{0}$ denote subgroup of those diffeomorphisms which induce the identity on $\pi_{1}(X)$. This group acts on $\mathscr{M}_{\Sigma}$.

The assignment

$$
(g, B) \mapsto \rho(X, \alpha, g)
$$

defines a function

$$
\rho(X): \mathscr{F}_{X} \times \mathscr{M}_{\Sigma} \rightarrow \mathbb{R}
$$

Theorems 3.2 and 5.1 say that $\rho(X)$ descends to a function on the quotient

$$
\rho(X): \chi\left(\pi_{1} X, U(n)\right) \times\left(\mathscr{M}_{\Sigma} / \mathscr{D}_{X}^{0}\right) \rightarrow \mathbb{R},
$$

where

$$
\chi\left(\pi_{1} X, U(n)\right)=\operatorname{Hom}\left(\pi_{1} X, U(n)\right) / \operatorname{conj} .=\mathscr{F}_{X} / \mathscr{G}_{X}
$$

with $\mathscr{G}_{X}$ the group of gauge transformations of $E \rightarrow X$. The quotient $\mathscr{D}_{X} / \mathscr{D}_{X}^{0}$ acts diagonally on $\chi\left(\pi_{1} X, U(n)\right) \times\left(\mathscr{M}_{\Sigma} / \mathscr{D}_{X}^{0}\right)$ and the function of (5.1) is invariant under this action.

\section{Dependence on the metric}

In this section we prove the following theorem which shows that the $\rho$ - and $m$-invariants depend on the choice of metric on the boundary. 


\section{Theorem 6.1}

(1) There exists a 3-manifold $Y$ with non-empty boundary, Riemannian metrics $g_{0}, g_{1}$ on $\partial Y$, and a representation $\alpha: \pi_{1} Y \rightarrow U(2)$ so that

$$
\rho\left(Y, \alpha, g_{0}\right) \neq \rho\left(Y, \alpha, g_{1}\right) .
$$

Examples exist with vanishing kernel of the tangential operator, i.e.

$$
\text { ker } A_{b} \cong H^{*}\left(\partial Y ; \mathbb{C}_{\alpha}^{2}\right)=0 .
$$

(2) There exist metrics $g_{0}$ and $g_{1}$ on the torus $T$ and 3-manifolds $X$ and $Y$ with boundary $T$ such that setting $V_{X}=\operatorname{image}\left(H^{*}(X ; \mathbb{C}) \rightarrow H^{*}(T ; \mathbb{C})\right)$ and $V_{Y}=\operatorname{image}\left(H^{*}(Y ; \mathbb{C}) \rightarrow H^{*}(T ; \mathbb{C})\right.$ ) (with $\theta$ the trivial connection),

$$
m\left(V_{X}, V_{Y}\right)_{\left(\theta, g_{0}\right)} \neq m\left(V_{X}, V_{Y}\right)_{\left(\theta, g_{1}\right)} .
$$

In the first statement of Theorem 6.1 the point of taking an example with $H^{*}\left(\partial Y ; \mathbb{C}_{\alpha}^{n}\right)=0$ is to emphasize that the metric dependence of the $\rho$-invariant is much more subtle than just being a consequence of the dependence of $m(V, W)$ on the metric.

To understand the significance of the second statement, observe that the choice of Riemannian metric on $\partial X=\Sigma$ enters into the definition of $m(V, W)$ only through the restriction of the induced $L^{2}$ metric on $L^{2}\left(\left.E\right|_{\Sigma}\right)$ to the harmonic forms ker $A_{b} \cong H^{*}\left(\Sigma ; \mathbb{C}^{n}\right)$. There are clearly many Riemannian metrics on $\Sigma$ which restrict to the same metric on the space of harmonic forms. It is perhaps at least intuitively clear that the invariant $m(V, W)$ of pairs of Lagrangian subspaces in a Hermitian symplectic space can vary as the inner product varies. But our argument shows more: the metrics we use are restrictions of $L^{2}$ metrics to the harmonic forms (i.e. the kernel of the tangential operator) and the Lagrangian subpaces we consider are of the form image $\left(H^{*}\left(X ; \mathbb{C}_{\alpha}^{n}\right)\right) \rightarrow$ $\left.H^{*}\left(\partial X ; \mathbb{C}_{\alpha}^{n}\right)\right)$. Notice that these Lagrangians $V_{X}$ are always graded direct sums; i.e. $V_{X}=\oplus_{i} V_{X}^{i}$ with $V_{X}^{i}=\operatorname{image}\left(H^{i}\left(X ; \mathbb{C}_{\alpha}^{n}\right) \rightarrow H^{i}\left(\partial X ; \mathbb{C}_{\alpha}^{n}\right)\right)$.

As an illuminating non-example the reader might consider the case when $\partial X$ is a $2 k$-sphere, and $\alpha$ is trivial. Then $\operatorname{ker} A_{b}=H^{*}\left(S^{2 k}\right)=H^{0}\left(S^{2 k}\right) \oplus H^{2 k}\left(S^{2 k}\right)$. Certainly one can find families of Riemannian metrics on $S^{2 k}$ so that the induced metric on the harmonic forms $H^{*}\left(S^{2 k}\right)$ varies (e.g. by scaling the metric) and from that it is not hard to produce a pair of Lagrangian subspaces $V, W \subset H^{*}\left(S^{2 k}\right)$ for which $m(V, W)_{(\theta, g)}$ varies with $g$. But, if $\alpha$ is the trivial representation on $\pi_{1} X$, then (for any such $X$ ) the subspace $V_{X}=$ image $H^{*}\left(X ; \mathbb{C}_{\alpha}^{n}\right) \rightarrow H^{*}\left(\partial X ; \mathbb{C}_{\alpha}^{n}\right)$ is just $H^{0}\left(S^{2 k}\right)$. Therefore, given a similar $Y, V_{X}=V_{Y}$ and so $\phi\left(V_{X}\right) \phi\left(V_{Y}\right)^{*}=I d$. This implies that the geometric invariant $m\left(V_{X}, V_{Y}\right)_{(\theta, g)}$ is independent of $g$ in this situation. 


\section{1 $\rho(X, \alpha, g)$ depends on $g$}

We begin with the proof of the first part of Theorem 6.1 by providing an explicit example which shows that $\rho(Y, \alpha, g)$ depends in general on the choice of Riemannian metric $g$ on the boundary $\partial Y$. We will show that there exists a 3-manifold $Y$ with boundary a torus, a non-abelian representation $\alpha: \pi_{1} Y \rightarrow$ $S U(2)$ with $H^{*}\left(\partial Y ; \mathbb{C}_{\alpha}^{2}\right)=0$, and Riemannian metrics $g_{0}$ and $g_{1}$ on the torus so that $\rho\left(Y, \alpha, g_{1}\right)-\rho\left(Y, \alpha, g_{0}\right)$ is non trivial. The metrics $g_{0}$ and $g_{1}$ can be taken to be flat.

The manifold $Y$ we take is the complement of the right-handed trefoil knot in $S^{3}$. The analysis of the space of $S U(2)$ representations of the fundamental groups of knot complements has a long history in the literature, starting with the beautiful article [27]. Details and proofs of most of the facts we use here can be found in [7].

The fundamental group of $Y$ is

$$
\pi_{1} Y=\left\langle x, y \mid x^{2}=y^{3}\right\rangle .
$$

The boundary of $Y$ is a torus, and the meridian $\mu$ and longitude $\lambda$ of $Y$ generate $\pi_{1}(\partial Y)=\mathbb{Z}^{2}$. They are given in this presentation of $\pi_{1} Y$ by

$$
\mu=x y^{-1} \text { and } \lambda=x^{2}\left(x y^{-1}\right)^{-6} .
$$

The space of conjugacy classes of non-abelian $S U(2)$ representations of $\pi_{1}(Y)$ is an open arc. Moreover, given any pair $(\phi, \psi)$ in the open line segment in $\mathbb{R}^{2}$

$$
\left\{\left(t,-6 t+\frac{1}{2}\right) \mid \frac{1}{12}<t<\frac{5}{12}\right\}
$$

there exists a unique conjugacy class of non-abelian $S U(2)$ representations of $\pi_{1}(Y)$ which satisfies

$$
\mu \mapsto\left(\begin{array}{cc}
e^{2 \pi i \phi} & 0 \\
0 & e^{-2 \pi i \phi}
\end{array}\right), \lambda \mapsto\left(\begin{array}{cc}
e^{2 \pi i \psi} & 0 \\
0 & e^{-2 \pi i \psi}
\end{array}\right) .
$$

Therefore, letting

$$
\left(\phi_{1}, \psi_{1}\right)=\left(\frac{1}{5},-\frac{7}{10}\right) \text { and }\left(\phi_{2}, \psi_{2}\right)=\left(\frac{2}{5},-\frac{19}{10}\right),
$$

we obtain two non-abelian representations $\alpha_{1}, \alpha_{2}: \pi_{1} Y \rightarrow S U(2)$ in the open $\operatorname{arc}$ of $(6.1)$.

Fix an identification of the boundary of $Y$ with the 2-torus $T=\mathbb{R}^{2} / \mathbb{Z}^{2}$ such that $\mu$ corresponds to the $x$-axis and $\lambda$ to the $y$-axis. Give $T$ the induced flat metric $g_{0}$. 
Consider the matrix

$$
f=\left(\begin{array}{ll}
1 & 3 \\
2 & 7
\end{array}\right) \in S L(2, \mathbb{Z})
$$

Then $f$ acts by right multiplication on $\mathbb{R}^{2}$ preserving the standard lattice $\mathbb{Z}^{2}$, and hence induces a diffeomorphism $f: T \rightarrow T$.

The first part of Theorem 6.1 follows from the next theorem.

Theorem 6.2 The difference $\rho\left(Y, \alpha_{1}, g_{0}\right)-\rho\left(Y, \alpha_{1}, f^{*}\left(g_{0}\right)\right)$ does not equal the difference $\rho\left(Y, \alpha_{2}, g_{0}\right)-\rho\left(Y, \alpha_{2}, f^{*}\left(g_{0}\right)\right)$. Hence the $\rho$-invariant for manifolds with boundary depends in general on the choice of Riemannian metric on the boundary, and moreover $\rho(Y, \alpha, g)-\rho\left(Y, \alpha, f^{*}(g)\right)$ is not a function of $f: \partial Y \rightarrow$ $\partial Y$ alone.

Proof Let $\hat{\alpha}_{i}: \pi_{1} T \rightarrow S U(2)$ denote the restrictions of $\alpha_{i}$ to $\pi_{1}(\partial Y)$. Let $g_{1}$ denote the pulled back metric $g_{1}=f^{*}\left(g_{0}\right)$.

Fix a Riemannian metric $\tilde{g}$ on $Y$ in product form near the boundary so that the restriction of $\tilde{g}$ to the boundary equals $g_{0}$.

Choose a smooth path $g_{t}$ of Riemannian metrics on $T$ from $g_{0}$ to $g_{1}$ which is stationary for $t \in[0, \epsilon]$ and $[1-\epsilon, 1]$. Then $g_{t}$ determines the metric $g_{t}+d t^{2}$ on $T \times[0,1]$.

Let $B_{1}$ be a flat connection on $Y$ with holonomy $\alpha_{1}$ and in cylindrical form $B=\pi^{*}\left(b_{1}\right)$ near $\partial Y$. Let $D_{B_{1}}$ denote the corresponding odd signature operator on $Y$. Then $D_{B_{1}}$ has an obvious extension to $Y \cup(T \times[0,1])$ by defining it to be the pullback of $b_{1}$ via the projection $T \times[0,1] \rightarrow T$. Similarly choose a flat connection $B_{2}$ with holonomy $\alpha_{2}$ and extend it to $T \times[0,1]$.

Lemma 6.3 $H^{*}\left(T ; \mathbb{C}_{\hat{\alpha}_{i}}^{2}\right)=0$.

Proof Applying the Fox calculus to the presentation

$$
\pi_{1}(\partial Y)=\left\langle\mu, \lambda \mid \mu \lambda \mu^{-1} \lambda^{-1}\right\rangle
$$

we conclude that $H^{*}\left(T ; \mathbb{C}_{\hat{\alpha}_{i}}^{2}\right)$ is the cohomology of the complex

$$
0 \rightarrow \mathbb{C}^{2} \stackrel{\partial_{0}}{\longrightarrow} \mathbb{C}^{2} \oplus \mathbb{C}^{2} \stackrel{\partial_{1}}{\longrightarrow} \mathbb{C}^{2} \rightarrow 0
$$

where

$$
\partial_{0}=\left(\begin{array}{ll}
\hat{\alpha}_{i}(\mu)-I & \hat{\alpha}_{i}(\lambda)-I
\end{array}\right) \text { and } \partial_{1}=\left(\begin{array}{l}
I-\hat{\alpha}_{i}(\lambda) \\
\hat{\alpha}_{i}(\mu)-I
\end{array}\right) .
$$

A simple computation using (6.2) and (6.3) shows that the cohomology of this complex vanishes. 
Continuing with the proof of Theorem 6.2, It follows from Corollary 4.2 that

$$
\begin{aligned}
\left(\rho\left(Y, \alpha_{1}, g_{1}\right)\right. & \left.-\rho\left(Y, \alpha_{1}, g_{0}\right)\right)-\left(\rho\left(Y, \alpha_{2}, g_{1}\right)-\rho\left(Y, \alpha_{2}, g_{0}\right)\right) \\
& =\eta\left(D_{B_{1}}, \Sigma \times[0,1]\right)-\eta\left(D_{B_{2}}, \Sigma \times[0,1]\right)
\end{aligned}
$$

(Note: Lemma 6.3 implies that ker $A_{b_{i}}=0$ so that there are no Lagrangian subspaces to specify in the $\eta$-invariants in (6.5).)

We will show that the right side of (6.5) is not an integer, from which Theorem 6.2 follows.

Let $M_{f}$ denote the mapping torus of $f$ :

$$
M_{f}=T \times[0,1] /(t, 0) \sim(f(t), 1) .
$$

The metric on $T \times[0,1]$ descends to a metric on $M_{f}$ since the gluing map $f:\left(T, g_{1}\right) \rightarrow\left(T, g_{0}\right)$ is an isometry.

Recalling that $\mu$ and $\lambda$ in $\pi_{1}(T)$ denote the two generators,

$$
\pi_{1}\left(M_{f}\right)=\left\langle\mu, \lambda, \tau \mid[\mu, \lambda]=1, \tau \mu \tau^{-1}=\mu \lambda^{2}, \tau \lambda \tau^{-1}=\mu^{3} \lambda^{7}\right\rangle .
$$

It follows that given a pair of real numbers $\phi, \psi$, the assignment

$$
\mu \mapsto\left(\begin{array}{cc}
e^{2 \pi i \phi} & 0 \\
0 & e^{-2 \pi i \phi}
\end{array}\right), \lambda \mapsto\left(\begin{array}{cc}
e^{2 \pi i \psi} & 0 \\
0 & e^{-2 \pi i \psi}
\end{array}\right) \text { and } \tau \mapsto\left(\begin{array}{cc}
0 & 1 \\
-1 & 0
\end{array}\right)
$$

determines a representation $\pi_{1}\left(M_{f}\right) \rightarrow S U(2)$ if and only if $e^{-2 \pi i \phi}=e^{2 \pi i(1 \phi+2 \psi)}$ and $e^{-2 \pi i \psi}=e^{2 \pi i(3 \phi+7 \psi)}$, i.e. if and only if

$$
(\phi \quad \psi)\left(f_{*}+\mathrm{Id}\right) \equiv 0 \quad(\bmod \mathbb{Z}) .
$$

Equation (6.7) holds for $\left(\phi_{1}, \psi_{1}\right)$ and $\left(\phi_{2}, \psi_{2}\right)$ as in (6.3).

Thus taking $\left(\phi_{1}, \psi_{1}\right)$ and $\left(\phi_{2}, \psi_{2}\right)$ in (6.6) we obtain two representations

$$
\beta_{j}: \pi_{1}\left(M_{f}\right) \rightarrow S U(2), j=1,2,
$$

with the property that their restrictions to the fiber $T \times\{0\}$ equal $\hat{\alpha}_{j}$.

View $M_{f}$ as the union of two cylinders $T \times[0,1] \cup T \times[0,1]$ using the gluing map $I d \cup f$. Give $M_{f}$ the product metric $g_{0}+d t^{2}$ on the first piece and $g_{t}+d t^{2}$ on the second. Equation (2.5) shows that

$$
\eta\left(D_{B_{j}}, M_{f}\right)=\eta\left(D_{B_{j}},\left(T \times[0,1], g_{0}+d t^{2}\right)\right)+\eta\left(D_{B_{j}},\left(T \times[0,1], g_{t}+d t^{2}\right)\right) .
$$

It follows from Corollary 4.2 that

$$
\eta\left(D_{B_{1}},\left(T \times[0,1], g_{0}+d t^{2}\right)\right)-\eta\left(D_{B_{2}},\left(T \times[0,1], g_{0}+d t^{2}\right)\right)=0
$$


(alternatively Lemma 7.1 of [26] shows directly that $\eta\left(D_{B_{j}},\left(T \times[0,1], g_{0}+\right.\right.$ $\left.\left.d t^{2}\right)\right)=0$ ).

Thus combining (6.8) for $j=1,2$ with (6.5) we obtain

$$
\begin{aligned}
\left(\rho\left(Y, \alpha_{1}, g_{1}\right)\right. & \left.-\rho\left(Y, \alpha_{1}, g_{0}\right)\right)-\left(\rho\left(Y, \alpha_{2}, g_{1}\right)-\rho\left(Y, \alpha_{2}, g_{0}\right)\right) \\
& =\eta\left(D_{B_{1}}, M_{f}\right)-\eta\left(D_{B_{2}}, M_{f}\right) \\
& =\rho\left(M_{f}, \beta_{1}\right)-\rho\left(M_{f}, \beta_{2}\right) .
\end{aligned}
$$

The last equality follows from the definitions of $\eta$ and $\rho$ for a closed manifold.

We have thus reduced the problem to showing that the difference of the $\rho$ invariants for $\beta_{1}$ and $\beta_{2}$ on the closed manifold $M_{f}$ is not an integer. On a closed manifold, the $\rho$-invariants and the Chern-Simons invariants are related by the Atiyah-Patodi-Singer theorem [2, 3]; the formula is (see [7, Sect. 5.3-5.5]):

$$
\begin{aligned}
\operatorname{SF}\left(D_{B_{t}}\right)=2( & \left.\operatorname{cs}\left(B_{1}\right)-\operatorname{cs}\left(B_{2}\right)\right) \\
& +\frac{1}{2}\left(\rho\left(M_{f}, \beta_{1}\right)-\rho\left(M_{f}, \beta_{2}\right)-\operatorname{dim} \operatorname{ker} D_{B_{1}}+\operatorname{dim} \operatorname{ker} D_{B_{2}}\right) .
\end{aligned}
$$

Here $\operatorname{SF}\left(D_{B_{t}}\right)$ denotes the spectral flow (an integer) of the family of self-adjoint elliptic operators $D_{B_{t}}$ where $B_{t}$ is any family of connections from $B_{1}$ to $B_{2}$. This implies

$$
\rho\left(M_{f}, \beta_{1}\right)-\rho\left(M_{f}, \beta_{2}\right) \equiv 4\left(\operatorname{cs}\left(B_{2}\right)-\operatorname{cs}\left(B_{1}\right)\right) \quad(\bmod \mathbb{Z}) .
$$

Theorem 5.6 of [19] calculates the Chern-Simons invariant mod $\mathbb{Z}$ of flat connections on $M_{f}$ in terms of the vector $(\phi, \psi)$ and the matrix $f$ : if $(m, n)=$ $(\phi, \psi)\left(\mathrm{I}+f^{-1}\right)$, then the Chern-Simons invariant of the flat connection with holonomy representation determined by $(\phi, \psi)$ equals $\phi n-\psi m \bmod \mathbb{Z}$.

Since $\left(\phi_{1}, \psi_{1}\right)\left(I+f^{-1}\right)=(3,-2)$ and $\left(\phi_{2}, \psi_{2}\right)\left(I+f^{-1}\right)=(7,-5)$ this gives

Hence

$$
c s\left(B_{1}\right) \equiv \frac{7}{10} \quad(\bmod \mathbb{Z}) \text { and } c s\left(B_{2}\right) \equiv \frac{3}{10} \quad(\bmod \mathbb{Z}) .
$$

$$
4\left(\operatorname{cs}\left(B_{1}\right)-\operatorname{cs}\left(B_{2}\right)\right) \equiv \frac{28}{10}-\frac{12}{10} \equiv \frac{3}{5} \quad(\bmod \mathbb{Z}) .
$$

Combining (6.9), (6.10), and (6.12) we see that

$$
\left(\rho\left(Y, \alpha_{1}, g_{1}\right)-\rho\left(Y, \alpha_{1}, g_{0}\right)\right)-\left(\rho\left(Y, \alpha_{2}, g_{1}\right)-\rho\left(Y, \alpha_{2}, g_{0}\right)\right) \neq 0,
$$

proving Theorem 6.2 and hence the first assertion of Theorem 6.1.

An interesting problem suggested by Corollary 4.2 and Theorem 6.2 is to find a description of the function $\chi(\Sigma, U(n)) \times \mathscr{D}_{\Sigma} / \mathscr{D}_{\Sigma}^{0} \rightarrow \mathbb{R}$ which takes $(\alpha, f)$ to $\rho\left(\Sigma \times[0,1], \alpha, g \cup f^{*}(g)\right)$ (i.e. the $\rho$-invariant of the cylinder with a fixed metric $g$ at $\Sigma \times\{0\}$ and $f^{*}(g)$ at $\left.\Sigma \times\{1\}\right)$. Theorem 6.2 implies that this map is non-trivial, and depends on $\alpha$. 


\section{$6.2 m\left(V_{X, \alpha}, V_{Y, \alpha}\right)_{(b, g)}$ depends on $g$}

We next prove the second assertion of Theorem 6.1.

Consider the 2-torus $T^{2}=S^{1} \times S^{1}$ with its standard oriented basis of 1-forms $\{d x, d y\}$. We consider these forms as sections of the trivial 1-dimensional complex bundle over $T^{2}$ endowed with the trivial connection.

For each $t>0$, give $T^{2}$ the Riemannian metric for which $\{d x, t d y\}$ is an orthonormal basis at each point. Letting $\hat{*}_{t}$ denote the corresponding Hodge *-operator we have

$$
\hat{*}_{t} d x=t d y, \quad \hat{*}_{t} d y=-\frac{1}{t} d x, \quad \hat{*}_{t} 1=t d x \wedge d y, \quad \text { and } \quad \hat{*}_{t}(d x \wedge d y)=\frac{1}{t} .
$$

Hence

$$
\gamma_{t}(d x)=t d y, \quad \gamma_{t}(d y)=-\frac{1}{t} d x, \quad \gamma_{t}(1)=t d x \wedge d y, \quad \text { and } \quad \gamma_{t}(d x \wedge d y)=-\frac{1}{t} .
$$

This defines the de Rham operator $A_{t}= \pm\left(\hat{*}_{t} d+d \hat{*}_{t}\right)$ as above.

The harmonic forms $\mathscr{H}_{t}^{*}=\operatorname{ker} A_{t}$ with respect to this metric are independent of $t$ as one can readily compute: the harmonic 0 -forms $\mathscr{H}^{0}$ are the constant functions, the harmonic 1-forms $\mathscr{H}^{1}$ are $a d x+b d y$ with $a, b$ constant, and the harmonic 2-forms $\mathscr{H}^{2}$ are $a d x \wedge d y$ with $a$ constant.

We can compute the $L^{2}$ inner product $\langle,\rangle_{t}$ restricted to the hamonic forms:

$$
\langle 1,1\rangle_{t}=\int_{T} 1 \wedge \hat{*}_{t} 1=\int_{T} t d x \wedge d y=4 \pi^{2} t .
$$

Similarly

$$
\begin{aligned}
& \langle d x, d x\rangle_{t}=4 \pi^{2} t, \quad\langle d x, d y\rangle_{t}=0, \quad\langle d y, d y\rangle_{t}=4 \pi^{2} / t \\
& \text { and }\langle d x \wedge d y, d x \wedge d y\rangle_{t}=4 \pi^{2} / t .
\end{aligned}
$$

The Hermitian symplectic space of harmonic forms $\left(\mathscr{H}^{*}, \gamma_{t},\langle,\rangle_{t}\right)$ is a direct sum $\left(\mathscr{H}^{0} \oplus \mathscr{H}^{2}\right) \oplus \mathscr{H}^{1}$ of two Hermitian symplectic spaces. Thus there is a corresponding splitting of the $\pm i$ eigenspaces of $\gamma_{t}$. One checks that the $\pm i$ eigenspaces of $\gamma_{t}$ acting on $\mathscr{H}^{*}$ are (with the obvious notation)

$$
\left(\mathscr{H}^{0} \oplus \mathscr{H}^{2}\right)^{ \pm}=\operatorname{span}\{1 \mp i t d x \wedge d y\}
$$

and

$$
\left(\mathscr{H}^{1}\right)^{ \pm}=\operatorname{span}\{d x \mp i t d y\}
$$


Suppose that $X$ is a compact 3 -manifold with $\partial X=T$. Then (taking $\mathbb{C}$ coefficients)

$$
\begin{aligned}
V_{X}= & \operatorname{image}\left(H^{*}(X) \rightarrow H^{*}(T)\right) \\
= & \operatorname{image}\left(H^{0}(X) \rightarrow H^{0}(T)\right) \oplus \operatorname{image}\left(H^{1}(X) \rightarrow H^{1}(T)\right) \oplus \\
& \oplus \operatorname{image}\left(H^{2}(X) \rightarrow H^{2}(T)\right) \\
= & H^{0}(T) \oplus \operatorname{image}\left(H^{1}(X) \rightarrow H^{1}(T)\right) \oplus 0 .
\end{aligned}
$$

Write $V_{X}^{1}$ for image $\left(H^{1}(X) \rightarrow H^{1}(T)\right)$. Similarly if $Y$ is another manifold with $\partial Y=T$ we have $V_{Y}=H^{0}(T) \oplus V_{Y}^{1}$ with $V_{Y}^{1}=\operatorname{image}\left(H^{1}(Y) \rightarrow H^{1}(T)\right)$.

Notice that since the coefficients are obtained by tensoring the integer cohomology with $\mathbb{C}$, there exist integers $a, b, A, B$ so that $V_{X}^{1}=\operatorname{span}\{a d x+b d y\}$ and $V_{Y}^{1}=\operatorname{span}\{A d x+B d y\}$. Moreover, given any pair of (not both zero) integers $(a, b)$ one can find a 3-manifold $X$ with $V_{X}^{1}=\operatorname{span}\{a d x+b d y\}$.

For example, take $X=S^{1} \times D^{2}$. By clearing denominators we may assume that $a$ and $b$ are relatively prime. Suppose that $p, q$ are integers satisfying $a p-b q=1$. Then there is a diffeomorphism $\partial X=S^{1} \times S^{1}$ to $T^{2}$ covered by the linear map $\mathbb{R}^{2} \rightarrow \mathbb{R}^{2}$ with matrix

$$
\left(\begin{array}{ll}
a & q \\
b & p
\end{array}\right)
$$

The closed 1-form $d x$ on $\partial X$ is identified with $a d x+b d y$ on $T^{2}$. Since $d x$ extends to $X$, this gives an example with $V_{X}^{1}=\operatorname{span}\{a d x+b d y\}$.

In terms of the $\pm i$ eigenspace decomposition of $\mathscr{H}^{*}$ one can easily check that

$$
\phi\left(V_{X}\right)=\phi^{0,2}\left(H^{0}(T)\right) \oplus \phi^{1}\left(V_{X}^{1}\right),
$$

where

$$
\phi^{0,2}\left(H^{0}(T)\right)(1-i t d x \wedge d y)=1+i t d x \wedge d y
$$

and

$$
\phi^{1}\left(V_{X}^{1}\right)(d x-i t d y)=\frac{i t a+b}{i t a-b}(d x+i t d y) .
$$

(See Equation (2.7) for the definition of the unitary map $\phi(V)$ associated to a Lagrangian subspace $V$.) These equations imply that

$$
\phi\left(V_{X}\right) \phi\left(V_{Y}\right)^{*}=\left(\begin{array}{cc}
1 & 0 \\
0 & \left(\frac{i t a+b}{i t a-b}\right)\left(\frac{i t A-B}{i t A+B}\right)
\end{array}\right) .
$$

Therefore (see (2.8))

$$
m\left(V_{X}, V_{Y}\right)_{\left(\tau, g_{t}\right)}=-\frac{1}{\pi i}\left(\pi i+\log \left(-\left(\frac{i t a+b}{i t a-b}\right)\left(\frac{i t A-B}{i t A+B}\right)\right)\right)+\operatorname{dim}\left(V_{X} \cap V_{Y}\right) .
$$


For example, taking $B=0$ this reduces to

$$
m\left(V_{X}, V_{Y}\right)_{\left(\theta, g_{t}\right)}=-1+\operatorname{dim}\left(V_{X} \cap V_{Y}\right)-\frac{1}{\pi i} \log \left(\frac{b+i t a}{b-i t a}\right) .
$$

But $\frac{b+i t a}{b-i t a}=\frac{(b+i t a)^{2}}{|b+i t a|^{2}}$ and so $\log \left(\frac{b+i t a}{b-i t a}\right)$ is equal to the argument of $(b+i t a)^{2}$ which varies non-trivially as $t$ varies provided both $a$ and $b$ are non-zero.

Thus we have given an example of a family of Riemannian metrics $g_{t}$ on the torus $T$ and shown how to find 3-manifolds $X$ and $Y$ so that (with respect to the trivial $U(1)$ representation $\tau) m\left(V_{X}, V_{Y}\right)_{\left(\theta, g_{t}\right)}$ varies non-trivially as $t$ is varied. This proves the second part of Theorem 6.1.

\section{An extension of the Farber-Levine-Weinberger theorem to manifolds with boundary}

Suppose that $F: M \rightarrow M^{\prime}$ is an orientation preserving homotopy equivalence of smooth compact manifolds. Then $F$ induces an isomorphism of fundamental groups, and hence a homeomorphism (in fact a real-analytic isomorphism)

$$
\operatorname{Hom}\left(\pi_{1}\left(M^{\prime}\right), U(n)\right) \stackrel{F^{*}}{\longrightarrow} \operatorname{Hom}\left(\pi_{1}(M), U(n)\right) .
$$

Taking the quotient by the action of conjugation eliminates the dependence on base points, and one obtains an identification (see Equation (5.2))

$$
\chi\left(\pi_{1}\left(M^{\prime}\right), U(n)\right)=\chi\left(\pi_{1}(M), U(n)\right) .
$$

If $M$ and $M^{\prime}$ are closed, then taking $\rho$-invariants defines functions (write $\pi=\pi_{1} M$ for convenience)

$$
\rho(M): \chi(\pi, U(n)) \rightarrow \mathbb{R} \text { and } \rho\left(M^{\prime}\right): \chi(\pi, U(n)) \rightarrow \mathbb{R} .
$$

In [14] M. Farber, J. Levine, and S. Weinberger proved the following remarkable theorem.

Theorem 7.1 (Farber-Levine, Weinberger) The difference

$$
\rho(M)-\rho\left(M^{\prime}\right): \chi(\pi, U(n)) \rightarrow \mathbb{R}
$$

factors through the set of path components of $\chi(\pi, U(n))$ and takes values in the rationals. Briefly, there is a commutative diagram

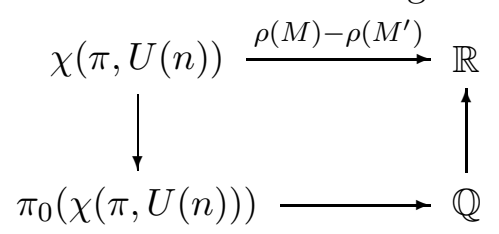

Algebraic 83 Geometric Topology, Volume 3 (2003) 
Moreover the difference $\rho(M)-\rho\left(M^{\prime}\right)$ vanishes on the path component containing the trivial representation.

Their proof has 3 ingredients. First Farber and Levine show that the difference $\rho(M)-\rho\left(M^{\prime}\right)$ modulo $\mathbb{Z}$ factors through the set of path components using the Atiyah-Patodi-Singer theorem and a computation of the index density. We will generalize this fact using the Dai-Freed theorem in Theorem 8.5.

Next they show that the "Z $\mathbb{Z}$ part", i.e. the spectral flow of the odd signature operator along a path of flat connections on a closed manifold, is a homotopy invariant which can be derived from a certain linking form. (A slightly different argument for this part was given in [23].)

Finally in an appendix Weinberger uses algebraic techniques to show that the difference is rational.

In this and the following section we will extend these results to manifolds with boundary, with respect to homotopy equivalences which restrict to diffeomorphisms on the boundary. (One cannot hope to prove a generalization for homotopy equivalences which do not behave nicely on the boundary; see Theorem 7.5.)

Suppose that $F: X \rightarrow X^{\prime}$ is a smooth map between compact manifolds which restricts to a diffeomorphism $f=\left.F\right|_{\partial X}: \partial X \cong \partial X^{\prime}$ on the boundary. Pulling back representations of $\pi_{1}\left(X^{\prime}\right)$ and Riemannian metrics on $\partial X^{\prime}$ induces a function (an analytic isomorphism if $F$ induces an isomorphism on fundamental groups):

$$
\chi\left(\pi_{1} X^{\prime}, U(n)\right) \times \mathscr{M}_{\partial X^{\prime}} \rightarrow \chi\left(\pi_{1} X, U(n)\right) \times \mathscr{M}_{\partial X} .
$$

In particular if $F$ is a homotopy equivalence we consider $\rho(X)$ and $\rho\left(X^{\prime}\right)$ as functions on the same space via this identification. Write $\pi$ for $\pi_{1} X$.

Theorem 7.2 Let $F: X \rightarrow X^{\prime}$ be a homotopy equivalence of compact manifolds which restricts to a diffeomorphism on the boundary. Then the difference

$$
\rho(X)-\rho\left(X^{\prime}\right): \chi(\pi, U(n)) \times \mathscr{M}_{\partial X} \rightarrow \mathbb{R}
$$

factors through $\pi_{0}(\chi(\pi, U(n))) \times\left(\mathscr{M}_{\partial X} / \mathscr{D}_{\partial X}^{0}\right)$ (where $\mathscr{D}_{\partial X}^{0}$ denotes the group of diffeomorphisms of $\partial X$ pseudo-isotopic to the identity) and takes values in the rational numbers.

In other words there is a commutative diagram

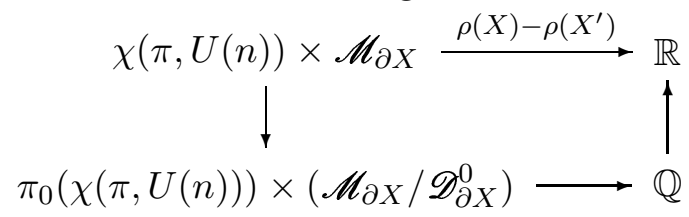

Algebraic 83 Geometric Topology, Volume 3 (2003) 
Moreover the difference $\rho(X)-\rho\left(X^{\prime}\right)$ vanishes on the path component of the trivial representation.

Proof We may asume, by homotoping $F$ slightly, that $F$ restricts to a diffeomorphim of collar neighborhoods of the boundary. Identify $\Sigma=\partial X$ with $\partial X^{\prime}$ via $f$, and fix a metric $g$ on $\Sigma$.

Let $\alpha$ be a $U(n)$ representation of $\pi_{1}(X)$. Consider the $(2 k+2)$-manifold $W=X \times[0,1]$. Then $\alpha$ clearly extends to $\pi_{1} W$. By smoothing the corners of $W$ we obtain a smooth manifold with boundary $\partial W=(-X) \cup_{\Sigma} X$. Since $W$ is a product, $\operatorname{Sign}(W)$ and $\operatorname{Sign}_{\alpha}(W)$ both vanish. The Atiyah-Patodi-Singer theorem then implies that $\rho(\partial W, \alpha)=0$.

(Alternatively, there is a direct spectral argument which shows the vanishing of $\rho(\partial W, \alpha)$ : the reflection which interchanges the two copies of $X$ in $\partial W=(-X) \cup_{\Sigma} X$ is orientation reversing, hence it anticommutes with the odd signature operator $D_{B}$. Thus the spectrum of $D_{B}$ is symmetric and so its $\eta$-invariant vanishes.)

The homotopy equivalence $F: X \rightarrow X^{\prime}$ induces a homotopy equivalence of closed manifolds

$$
\operatorname{Id} \cup F: \partial W=(-X) \cup_{\Sigma} X \rightarrow(-X) \cup_{\Sigma} X^{\prime} .
$$

The Farber-Levine-Weinberger theorem then implies that

$$
\rho\left((-X) \cup_{\Sigma} X^{\prime}, \alpha\right)=\rho\left((-X) \cup_{\Sigma} X^{\prime}, \alpha\right)-\rho(\partial W, \alpha)=r \in \mathbb{Q}
$$

for some rational number $r$ which depends only on the path component of $\alpha$ in $\chi\left(\pi_{1}(W), U(n)\right)=\chi(\pi, U(n))$.

Using Theorem 3.2 , part 4 we conclude that

$$
\rho\left((-X) \cup_{\Sigma} X^{\prime}, \alpha\right)=\rho\left(X^{\prime}, \alpha, g\right)+\rho\left(-X, \alpha, g, \gamma\left(V_{X^{\prime}, \alpha}\right), \gamma\left(V_{X^{\prime}, \tau}\right)\right)
$$

and

$$
\rho\left((-X) \cup_{\Sigma} X, \alpha\right)=\rho(X, \alpha, g)+\rho\left(-X, \alpha, g, \gamma\left(V_{X, \alpha}\right), \gamma\left(V_{X, \tau}\right)\right) .
$$

The commutative diagram (with any coefficients)

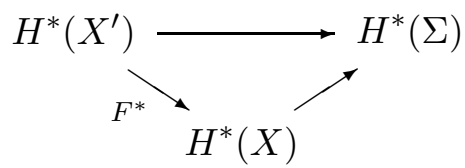

shows that $V_{X^{\prime}, \alpha}=V_{X, \alpha}$ and $V_{X^{\prime}, \tau}=V_{X, \tau}$. Therefore,

$$
\rho\left(-X, \alpha, g, \gamma\left(V_{X, \alpha}\right), \gamma\left(V_{X, \tau}\right)\right)=\rho\left(-X, \alpha, g, \gamma\left(V_{X^{\prime}, \alpha}\right), \gamma\left(V_{X^{\prime}, \tau}\right)\right) .
$$


Taking the difference of (7.2) and (7.3) and using (7.1) and (7.4) we conclude that

$$
\rho\left(X^{\prime}, \alpha, g\right)-\rho(X, \alpha, g)=\rho\left((-X) \cup_{\Sigma} X^{\prime}, \alpha\right)=r \in \mathbb{Q}
$$

for an $r$ that depends only on the path component of $\alpha$ in $\chi(\pi, U(n))$. Notice that if $\alpha$ is trivial, then $\rho(X, \alpha, g)=0$.

The fact that $\rho(X, \alpha, g)$ depends only on the pseudo-isotopy class of $g$ follows from Corollary 5.2.

The reader should keep in mind that the $\rho$-invariants in the context of Theorems 7.1 and 7.2 are not continuous in $\alpha$. This is because eigenvalues of the odd signature operator can become zero, or change sign, as $\alpha$ is varies. Thus what is being asserted in these theorems is that the discontinuities of $\rho$ are homotopy invariants, provided the homotopy equivalence restricts to a diffeomorphism on the boundary.

We formalize and extend this remark in Theorem 7.4 below which shows that the spectral flow of the odd signature operator coupled to a path of flat connections on a manifold with boundary is a homotopy invariant. For a closed manifold this is the main result of [14], and the principal ingredient in the proof of Theorem 7.1. Partial results for manifolds with boundary were obtained in a series of articles by E. Klassen and the first author, including [24, 22, 25] as well as in the articles $[21,7]$ which also contain applications of these ideas to calculations of Floer homology, $S U(3)$ Casson invariants, and TQFT.

Consider, then, a path $B_{t}, t \in[0,1]$ of flat $U(n)$ connections, in cylindrical form near the boundary, on a compact smooth manifold $X$ with boundary $\partial X$. These give a path $D_{B_{t}}$ of odd signature operators in the form $D_{B_{t}}=\gamma\left(\frac{\partial}{\partial x}+A_{b_{t}}\right)$ on a collar neighborhood of $\partial X$.

To obtain a path of self-adjoint operators on $X$ whose kernels have a topological meaning we assume that we are given a continuous path of APS boundary conditions. Precisely, we assume that we are given a continuous path $P(t)$ of Lagrangian subspaces of $L^{2}\left(\left.E\right|_{\partial X}\right)$ so that for each $t$ there exists a (finitedimensional) subspace $W(t) \subset \operatorname{ker} A_{b_{t}}$ for which

$$
P(t)=F_{b_{t}}^{+}(t) \oplus W(t) .
$$

The path of operators $D_{B_{t}, P(t)}$ (recall this means $D_{B_{t}}$ with the boundary condition given by $P(t) u=0$ ) is a path of self-adjoint discrete operators (see Section 2, [26], and in particular [8, Sec. 3]) and hence has a spectral flow $\operatorname{SF}\left(X, D_{B_{t}, P(t)}\right)_{t \in[0,1]} \in \mathbb{Z}$. We use the $(-\epsilon,-\epsilon)$ convention for spectral flow; 
this implies that the spectral flow is additive with respect to composition of paths.

To ensure that the spectral flow of the resulting path of self-adjoint operators $D_{B_{t}, P(t)}$ is a topological invariant we furthermore assume that

$$
P(0)=F_{b_{0}}^{+} \oplus V_{X, \alpha_{0}} \text { and } P(1)=F_{b_{1}}^{+} \oplus V_{X, \alpha_{1}} .
$$

As before $F_{b_{t}}^{+}(t)$ denotes the positive eigenspan of $A_{b_{t}}$. The following lemma shows that such a path can always be found, and that the resulting spectral flow is independent of the choice of the Riemannian metric. What makes the proof of Lemma 7.3 tricky is that we do not assume the kernels of the family $A_{b_{t}}$ have constant dimension.

Lemma 7.3 Suppose that $B_{0}$ and $B_{1}$ are two flat $U(n)$ connections on $X$ whose holonomies $\alpha_{0}, \alpha_{1}$ lie in the same path component of $\chi\left(\pi_{1} X, U(n)\right)$.

Then, perhaps after gauge transforming $B_{1}$, there is a continuous piecewise smooth path $B_{t}$ of flat $U(n)$ connections joining them and a continuous piecewise smooth path $P(t)=F_{b_{t}}^{+}(t) \oplus W(t)$ of self-adjoint APS boundary conditions for the corresponding odd signature operators, with $P(0)=F_{b_{0}}^{+} \oplus V_{X, \alpha_{0}}$ and $P(1)=F_{b_{1}}^{+} \oplus V_{X, \alpha_{1}}$. In fact this path can be taken to be piecewise real analytic.

Moreover, the spectral flow of the path $D_{B_{t}, P(t)}$ of self-adjoint operators, $\mathrm{SF}\left(D_{B_{t}, P(t)}\right) \in \mathbb{Z}$, depends only on the path $\alpha: I \rightarrow \chi\left(\pi_{1} X, U(n)\right)$ of holonomies of $B_{t}$ and the choice of $W(t)$. In particular it is independent of the choice of Riemannian metric on $X$ (and $\partial X$ ).

Proof We prove the last assertion first. Note that the kernel of $D_{B_{t}, P(t)}$ is isomorphic to

$$
\text { image }\left(H^{*}\left(X, \partial X ; \mathbb{C}_{\alpha_{t}}^{n}\right) \rightarrow H^{*}\left(X ; \mathbb{C}_{\alpha_{t}}^{n}\right)\right) \oplus\left(W(t) \cap \gamma\left(V_{X, \alpha_{t}}\right)\right)
$$

by (2.3) (as usual $\alpha_{t}$ denotes the holonomy representation of $B_{t}$ ). This is not quite a homotopy invariant since it is possible that by varying the metric one could change the intersection of $W(t)$ (which is metric independent) with $\gamma\left(V_{X, \alpha_{t}}\right)$ (which can vary with the metric since $\gamma$ does). However, at the endpoints $W(i)=V_{X, \alpha_{i}}, i=0,1$, and since $\gamma(L)=L^{\perp}$ for any Lagrangian, $W(i) \cap \gamma\left(V_{X, \alpha_{i}}\right)=0$ for $i=0,1$. Thus the dimension of the kernel of $D_{B_{i}, P(i)}$ is independent of the choice of Riemannian metric on $X$ and $\partial X$.

Varying the Riemannian metric on $X$ varies the path $D_{B_{t}, P(t)}$ continuously in the space of self-adjoint operators $([8$, Sec. 3$])$. Moreover, since the dimensions 
of the kernels at the endpoints are homotopy invariants it follows that varying the metric does not change the spectral flow.

Suppose that $B_{t}^{\prime}$ is another continuous path of flat connections with the same holonomy as $B_{t}$. Then one can find a continuous path of gauge transformations $g_{t}$ so that $g_{t} \cdot B_{t}=B_{t}^{\prime}$. It is then straightforward to use $g_{t}$ to define unitary transformations which conjugate the operators $D_{B_{t}, P(t)}$ to $D_{B_{t}^{\prime}, P^{\prime}(t)}$, and so the spectral flows of the two paths coincide. We leave the details to the reader

We turn to the problem of constructing the paths $B_{t}$ and $W(t)$. Using the main result of [15] one can find a piecewise real analytic path $B_{t}$ of flat connections joining $B_{0}$ to $B_{1}^{\prime}$, where $B_{1}^{\prime}$ is a flat connection gauge equivalent to $B_{1}$. Indeed we can pick a piecewise analytic path $\alpha_{t}$ in $\chi\left(\pi_{1} X, U(n)\right)$ from $\alpha_{0}$ to $\alpha_{1}$, and the main theorem of [15] shows that one can find a finite covering of the interval $[0,1]$ and analytic paths of flat connections in each subinterval with the corresponding holonomy.

By relabeling assume that $B_{1}=B_{1}^{\prime}$. Then by subdividing further if necessary, the interval $[0,1]$ can broken down into subintervals $\left[t_{i}, t_{i+1}\right]$ so that on each such subinterval:

(1) The path $B_{t}$ is real-analytic.

(2) The kernel of the tangential operator, ker $A_{b_{t}}$, has constant dimension on the interior of the interval.

The reason why the second condition can be met is that the subspaces

$$
S_{k}:=\left\{\alpha \in \chi\left(\pi_{1} X, U(n)\right) \mid \operatorname{dim} H^{*}\left(\partial X, \mathbb{C}_{\alpha}^{n}\right) \leq k\right\} \subset \chi(\pi, U(n))
$$

form a real-analytic subvariety and so an analytic path intersects it in a finite number of points.

For convenience, reparametrize the path so that on alternate intervals the path is constant, i.e. $B_{t}$ is constant on $\left[0, t_{1}\right],\left[t_{2}, t_{3}\right], \cdots,\left[t_{2 m}, t_{2 m+1}\right], \cdots,\left[t_{2 \ell}, 1\right]$.

We now construct the path $W(t)$.

The $W(i)$ have already been chosen at the endpoints: we take $W(0)=V_{X, \alpha_{0}} \subset$ $H^{*}\left(\Sigma ; \mathbb{C}_{\alpha_{0}}^{n}\right)$ and $W(1)=V_{X, \alpha_{1}} \subset H^{*}\left(\Sigma ; \mathbb{C}_{\alpha_{1}}^{n}\right)$.

Next, on each interval $\left[t_{2 m-1}, t_{2 m}\right]$, by the Kato selection lemma [18] we can find analytically varying eigenvectors $\psi_{j}(t)$ with analytically varying eigenvalues $\mu_{j}(t)$ for $j \in\{ \pm 1, \pm 2, \cdots\}$.

The set $\left\{\psi_{j}\right\}$ can be partitioned into the finite subset

$$
K:=\left\{\psi_{j} \mid \mu_{j}(t)=0 \text { for all } t \in\left[t_{2 m-1}, t_{2 m}\right]\right\}
$$


and its complement. Moreover, by relabeling we may assume that

$$
K=\left\{\psi_{j} \mid j= \pm 1, \pm 2, \cdots, \pm \ell\right\} .
$$

We further assume, by changing bases, that $\gamma\left(\psi_{j}(t)\right)=\psi_{-j}(t)$ for $j=1, \cdots, \ell$ and $\psi_{j}(t) \perp \psi_{k}(t)$ for $j, k \in\{ \pm 1, \cdots \pm \ell\}$ for all $t \in\left[t_{2 m-1}, t_{2 m}\right]$. This is possible because

$$
S(t):=\operatorname{span}\left\{\psi_{j}(t) \mid j= \pm 1, \cdots \pm \ell\right\}
$$

is preserved by $\gamma$ for each $t \in\left[t_{2 m-1}, t_{2 m}\right]$ and hence is an analytically varying family of Hermitian symplectic spaces (note that $S(t)=\operatorname{ker} A_{b_{t}}$ for $t$ in the interior of $\left.\left[t_{2 m-1}, t_{2 m}\right]\right)$. The spaces $S(t)$ contain an analytically varing family of Lagrangian subspaces

$$
L(t):=\operatorname{span}\left\{\psi_{j}(t) \mid j=1, \cdots, \ell\right\} \subset S(t) .
$$

Then define $W(t)$ on $\left[t_{2 m-1}, t_{2 m}\right]$ as follows.

(1) For $t_{2 m-1}<t<t_{2 m}$ take

$$
W(t)=L(t)
$$

(2) For $t=t_{2 m-1}$,

$$
\begin{aligned}
& W\left(t_{2 m-1}\right)=L\left(t_{2 m-1}\right) \oplus \\
& \oplus \operatorname{span}\left\{\psi_{j}\left(t_{2 m-1}\right) \mid \mu_{j}\left(t_{2 m-1}\right)=0 \text { and } \mu_{j}(t)>0 \text { for } t_{2 m-1}<t<t_{2 m}\right\} .
\end{aligned}
$$

Briefly, $W\left(t_{2 m-1}\right)$ is the span of $\lim _{t \rightarrow t_{2 m-1}^{+}} L(t)$ and those zero eigenvectors that deform to positive eigenvectors for $t>t_{2 m-1}$.

(3) For $t=t_{2 m}$,

$$
\begin{aligned}
& W\left(t_{2 m}\right)=L\left(t_{2 m}\right) \oplus \\
& \oplus \operatorname{span}\left\{\psi_{j}\left(t_{2 m}\right) \mid \mu_{j}\left(t_{2 m}\right)=0 \text { and } \mu_{j}(t)>0 \text { for } t_{2 m-1}<t<t_{2 m}\right\} .
\end{aligned}
$$

Thus $W\left(t_{2 m}\right)$ is the span of $\lim _{t \rightarrow t_{2 m-1}^{-}} L(t)$ and those zero eigenvectors that deform to positive eigenvectors for $t<t_{2 m}$.

By construction, $P(t)=F_{b_{t}}^{+}(t) \oplus W(t)$ is smooth (even analytic) on the interval $\left[t_{2 m-1}, t_{2 m}\right]$. That the $W(t)$ are Lagrangian is immediate except possibly at the endpoints. But at the endpoint $t_{2 m-1}$ the decomposition of ker $A_{b_{t_{2 m-1}}}$ into the nullvectors that "stay null" and those that deform into non-zero eigenvectors is a decomposition as a symplectic direct sum, and in the summand corresponding to the eigenvectors that deform into non-zero eigenvectors the subspace of those that deform into positive eigenvectors is Lagrangian. Thus $W\left(t_{2 m-1}\right)$ is a direct sum of Lagrangian subspaces, and hence is Lagrangian. A similar argument applies to $W\left(t_{2 m}\right)$. 
It remains to define $W(t)$ on the intervals $\left[t_{2 m}, t_{2 m+1}\right]$. On these intervals the connection $B_{t}$ is constant. Hence the symplectic space ker $A_{b_{t}}$ is constant also. The Lagrangians $W\left(t_{2 m}\right)$ and $W\left(t_{2 m+1}\right)$ have already been defined, so just pick some smooth path $W(t)$ interpolating between these two. With care this path can be chosen to be analytic. Clearly the path $P(t)=F_{b_{t}}^{+}(t) \oplus W(t)$ is smooth on this interval.

The following theorem says that the spectral flow, which by Lemma 7.3 is a diffeomorphism invariant, is in fact an invariant of homotopy equivalences which restrict to diffeomorphisms on the boundary.

Theorem 7.4 Suppose that $F: X^{\prime} \rightarrow X$ is a homotopy equivalence which restricts to a diffeomorphism $f=\left.F\right|_{\partial X^{\prime}}: \partial X^{\prime} \rightarrow \partial X$. Assume that $B_{t}$ is a continuous, piecewise smooth path of flat $U(n)$ connections on $E \rightarrow X$. Use $F$ to pull back the path $B_{t}$ to a path of flat connections $B_{t}^{\prime}$ on $X^{\prime}$ and to identify $\partial X$ with $\partial X^{\prime}$, and choose a path $P(t)$ of APS boundary conditions as in Lemma 7.3.

Then

$$
\mathrm{SF}\left(X, D_{B_{t}, P(t)}\right)_{t \in[0,1]}=\mathrm{SF}\left(X^{\prime}, D_{B_{t}^{\prime}, P(t)}\right)_{t \in[0,1]} .
$$

Proof Since spectral flow is additive with respect to composition of paths, we may assume that the path $D_{B_{t}, P(t)}$ is a smooth path of self-adjoint operators. Denote this path by $D_{t}$; thus $\eta\left(D_{t}\right)=\eta\left(D_{B_{t}, W(t)}, X\right)$.

Theorem 3.2, part 1 and the definitions imply that

$$
\begin{aligned}
\eta\left(D_{s}\right)-\eta\left(D_{0}\right)= & \eta\left(D_{B_{s}, V_{X, \alpha_{s}}}, X\right)+m\left(\gamma\left(V_{X, \alpha_{s}}\right), W(s)\right)_{\left(\alpha_{s}, g\right)} \\
& -\eta\left(D_{B_{0}, V_{X, \alpha_{0}}}, X\right)-m\left(\gamma\left(V_{X, \alpha_{0}}\right), W(0)\right)_{\left(\alpha_{0}, g\right)} \\
= & \rho\left(X, D_{B_{s}}, g\right)+m\left(\gamma\left(V_{X, \alpha_{s}}\right), W(s)\right)_{\left(\alpha_{s}, g\right)} \\
& -\rho\left(X, D_{B_{0}}, g\right)-m\left(\gamma\left(V_{X, \alpha_{0}}\right), W(0)\right)_{\left(\alpha_{0}, g\right)},
\end{aligned}
$$

where $\alpha_{t}$ denotes the holonomy of $B_{t}$ and $g$ is the metric on $\partial X$.

The reduction of the $\eta$-invariants $\eta\left(D_{t}\right)$ modulo $\mathbb{Z}$ is smooth in $t$. Combining the formula (see e.g. [26, Lemma 3.4])

$$
\eta\left(D_{s}\right)-\eta\left(D_{0}\right)=2 \mathrm{SF}\left(D_{t}\right)_{t \in[0, s]}-\left(\operatorname{dim} \operatorname{ker} D_{s}-\operatorname{dim} \operatorname{ker} D_{0}\right)+\int_{0}^{s} \frac{d \eta\left(D_{t}\right)}{d t} d t
$$

with (7.5) yields

$$
\begin{aligned}
\rho\left(X, \alpha_{s}, g\right)-\rho\left(X, \alpha_{0}, g\right) & \\
& +m\left(\gamma\left(V_{X, \alpha_{s}}\right), W(s)\right)_{\left(\alpha_{s}, g\right)}-m\left(\gamma\left(V_{X, \alpha_{0}}\right), W(0)\right)_{\left(\alpha_{0}, g\right)} \\
= & 2 \operatorname{SF}\left(D_{t}\right)_{t \in[0, s]}-\left(\operatorname{dim} \operatorname{ker} D_{s}-\operatorname{dim} \operatorname{ker} D_{0}\right)+\int_{0}^{s} \frac{d \eta\left(D_{t}\right)}{d t} d t .
\end{aligned}
$$


Similarly

$$
\begin{aligned}
\rho\left(X^{\prime}, \alpha_{s}, g\right)-\rho\left(X^{\prime}, \alpha_{0}, g\right) & \\
& +m\left(\gamma\left(V_{X^{\prime}, \alpha_{s}}\right), W(s)\right)_{\left(\alpha_{s}, g\right)}-m\left(\gamma\left(V_{X^{\prime}, \alpha_{0}}\right), W(0)\right)_{\left(\alpha_{0}, g\right)} \\
= & 2 \operatorname{SF}\left(D_{t}^{\prime}\right)_{t \in[0, s]}-\left(\operatorname{dim} \operatorname{ker} D_{s}^{\prime}-\operatorname{dim} \operatorname{ker} D_{0}^{\prime}\right)+\int_{0}^{s} \frac{d \eta\left(D_{t}^{\prime}\right)}{d t} d t .
\end{aligned}
$$

where $D_{t}^{\prime}$ denotes the odd signature operator on $X^{\prime}$ coupled to the path $B_{t}^{\prime}$ with boundary conditions given by the projection to $P(t)$.

Taking the difference of (7.7) and (7.8) and using Theorem 7.2, the fact that $V_{X, \alpha_{s}}=V_{X^{\prime}, \alpha_{s}}$, and (2.3) one concludes

$$
2 \mathrm{SF}\left(D_{t}\right)_{t \in[0, s]}-2 \mathrm{SF}\left(D_{t}^{\prime}\right)_{t \in[0, s]}=\int_{0}^{s} \frac{d \eta\left(D_{t}^{\prime}\right)}{d t} d t-\int_{0}^{s} \frac{d \eta\left(D_{t}\right)}{d t} d t .
$$

The left side is an integer-valued function of $s$. The right side is a smooth real-valued function of $s$ which vanishes at $s=0$. Thus both sides vanish for all $s$ and so

$$
\mathrm{SF}\left(D_{t}\right)_{t \in[0, s]}=\mathrm{SF}\left(D_{t}^{\prime}\right)_{t \in[0, s]}
$$

as desired.

Theorems 7.2 and 7.4 do not hold without some assumption about the restriction of the homotopy equivalence to the boundary. Here is an an example. Consider the complements $X=S^{3}-\operatorname{nbd}(K)$ of the Square knot $K$ and $X^{\prime}=S^{3}-\operatorname{nbd}\left(K^{\prime}\right)$ of the Granny knot $K^{\prime}$. (The Square knot is a connected sum of a right-handed Trefoil knot and a left-handed Trefoil knot. The Granny knot is the connected sum of two right-handed Trefoil knots.) The spaces $X$ and $X^{\prime}$ have isomorphic fundamental groups and are aspherical, and so they are homotopy equivalent (see e.g. [34] and [17]). Each has a torus boundary. But there does not exist a homotopy equivalence which restricts to a diffeomorphism (or even a homotopy equivalence) on the boundary. This follows from Waldhausen's theorem [35] (it also follows from the following argument and Theorem 7.4).

Since $H_{1}(X ; \mathbb{Z})=\mathbb{Z}=H_{1}\left(X^{\prime} ; \mathbb{Z}\right)$, the $U(1)$ character variety of $\pi_{1} X=\pi_{1} X^{\prime}$ is a circle, parameterized by the image $z=e^{i x}$ of the generator of the first homology. Fix a generator $\mu \in H_{1}(X ; \mathbb{Z})$. Let $F: X \rightarrow X^{\prime}$ be a homotopy equivalence. Let $\alpha_{z}: \pi_{1}(X) \rightarrow U(1)$ be the representation which takes $\mu$ to $z=e^{i x}$. Let $B_{z}$ be a path of flat connections on $X$ with holonomy $\alpha_{z}$ and restriction $b_{z}$ to the boundary. Let $B_{z}^{\prime}=F^{*}\left(B_{z}\right)$. 
Theorem 7.5 The 3-manifolds $X$ and $X^{\prime}$ are homotopy equivalent, have diffeomorphic boundaries, but for no choice of metrics $g, g^{\prime}$ on $\partial X, \partial X^{\prime}$ is $\rho(X, \alpha, g)$ equal to $\rho\left(X^{\prime}, \alpha, g^{\prime}\right)$ for all $\alpha \in \chi\left(\pi_{1} X, U(1)\right)$.

Proof Suppose that $g$ is a metric on $\partial X$ and $g^{\prime}$ a metric on $\partial X^{\prime}$.

Lemma 6.3 shows that for $z \neq 1, H^{*}\left(\partial X ; \mathbb{C}_{\alpha_{z}}\right)=\operatorname{ker} A_{b_{z}}=0$. Thus the boundary conditions given by the projection to the positive eigenspan $F_{b_{z}, g}^{+}$of $A_{b_{z}}$ are self-adjoint (for $z \neq 1$ ) and vary smoothly in $z$. Similarly for $X^{\prime}$.

The kernel of the operator $D_{B_{z}}$ with these boundary conditions is zero except for those $z$ which are roots of the Alexander polynomial of the Granny knot $K$. Moreover, the spectral flow as $z$ moves through the root is given by the change in the Levine-Tristram signature (see e.g. [30]) of $K$. These facts are proven e.g. in [21].

Since the Square and Granny knots have isomorphic groups, their Alexander polynomials are the same, namely $\left(z^{2}-z+1\right)^{2}$. Thus eigenvalues of $D_{B_{z}}$ and $D_{B_{z}^{\prime}}$ cross zero for the same values of $z$, namely $e^{2 \pi i / 6}$ and $e^{5 \pi i / 6}$.

The spectral flow through these values of $z$ is different for $X$ and $X^{\prime}$. Indeed, the Square knot is slice and hence has vanishing Levine-Tristram signatures; thus $\operatorname{SF}\left(D_{B_{z}}\right)$ is zero. But the Levine-Tristram signatures for the Granny knot are non-trivial (they detect the non-sliceness of the Granny knot). Thus if $J$ is a small interval in $U(1)$ containing a root of the Alexander polynomial (to be explicit, we can take $\left.J=\exp \left(2 \pi i\left[\frac{1}{6}-\epsilon, \frac{1}{6}+\epsilon\right]\right)\right)$

$$
\operatorname{SF}\left(X, D_{B_{z}, F_{b_{z}}^{+}}\right)_{z \in J}=0 \text { and } \operatorname{SF}\left(X, D_{B_{z}, F_{b_{z}^{\prime}}^{+}}\right)_{z \in J}=2 .
$$

The reduction of $\rho\left(X, \alpha_{z}, g\right)$ to $\mathbb{R} / \mathbb{Z}$ is continuous in $z$. Since the integer jumps of $\rho\left(X, \alpha_{z}, g\right)$ as $z$ varies are given by the spectral flow (see (7.6)), it follows that for some $z$ near $e^{2 \pi i / 6}$,

$$
\rho\left(X, \alpha_{z}, g\right) \neq \rho\left(X^{\prime}, \alpha_{z}, g^{\prime}\right) .
$$

\section{Determinant bundles and variation of the $\rho$-invariant $\bmod \mathbb{Z}$ on manifolds with boundary}

As before let $X$ be a compact odd-dimensional manifold with boundary $\Sigma$. We are given a bundle $E \rightarrow X$ and a flat connection $B$ on $E$ in cylindrical form $b+\frac{\partial}{\partial u}$ near the boundary, and a Riemannian metric $\tilde{g}$ on $X$ in cylindrical form $g+d u^{2}$ near the boundary, for some Riemannian metric $g$ on $\Sigma$. 
Recall that the determinant line of the operator $A_{b}$ is the (complex) vector space

$$
\operatorname{det}\left(\operatorname{ker} A_{b}\right)=\left(\operatorname{det} \operatorname{ker} A_{b}^{+}\right)^{-1} \otimes \operatorname{det} \operatorname{ker} A_{b}^{-},
$$

where $\operatorname{ker} A_{b}^{ \pm}:=\operatorname{ker} A_{b} \cap \operatorname{ker}(\gamma \mp i)$, i.e. ker $A_{b}$ is considered as a $\mathbb{Z}_{2}$-graded vector space with grading operator $-i \gamma$. For details about graded determinant lines we refer to $[13$, Sec. II].

Given a Lagrangian $W \subset$ ker $A_{b}$, it can be written as the graph of the unitary isomorphism $\phi(W)$ : $\operatorname{ker} A_{b}^{+} \rightarrow \operatorname{ker} A_{b}^{-}$. Thus $\operatorname{det}(\phi(W))$ is naturally an element of $\operatorname{det}\left(\operatorname{ker} A_{b}\right)$. Theorem 3.2 shows that

$$
e^{2 \pi i \tilde{\eta}\left(D_{B, W}, X\right)} \operatorname{det}(\phi(W))^{-1}=e^{2 \pi i \tilde{\eta}\left(D_{B, V_{X, \alpha}}, X\right)} \operatorname{det}\left(\phi\left(V_{X, \alpha}\right)\right)^{-1} .
$$

Hence the expression $s(X, \alpha, g):=e^{2 \pi i \tilde{\eta}\left(D_{B, W}, X\right)} \operatorname{det}(\phi(W))^{-1}$ is independent of $W$, and so well-defines an element in $\left(\operatorname{det} \operatorname{ker} A_{b}\right)^{-1}$. As a consequence, we obtain a well-defined element

$$
\begin{aligned}
& e^{2 \pi i \tilde{\eta}\left(D_{B, W_{\alpha}}, X\right)} e^{-2 \pi i \tilde{\eta}\left(D_{\Theta, W_{\tau}}, X\right)} \operatorname{det}\left(\phi\left(W_{\alpha}\right)\right)^{-1} \otimes \operatorname{det}\left(\phi\left(W_{\tau}\right)\right) \\
& \in\left(\operatorname{det} \operatorname{ker} A_{b}\right)^{-1} \otimes \operatorname{det} \operatorname{ker} A_{\theta},
\end{aligned}
$$

independent of the choice of $W_{\alpha}$ and $W_{\tau}$. Here $\Theta$ denotes the trivial connection on $\mathbb{C}^{n} \times X \rightarrow X$ and $\theta$ its restriction to $\Sigma$. By slight abuse of notation we will denote the element $s(X, \alpha, g) s(X, \tau, g)^{-1}$ of $\left(\operatorname{det} \operatorname{ker} A_{b}\right)^{-1} \otimes \operatorname{det} \operatorname{ker} A_{\theta}$ given in (8.2) by $e^{\pi i \rho(X, \alpha, g)}$.

For the spin Dirac operator the fact that the expression on the left hand side of (8.1) gives a well-defined element of the inverse determinant line was observed first by X. Dai and D. Freed [13, Sec. I]. As pointed out in [13] the result easily transfers to general Dirac operators. Due to the exponentiation, (8.1) does not need the full strength of Theorem 3.2. As in [13] it can be derived already from [29] where the dependence of the $\bmod \mathbb{Z}$ reduced $\eta$-invariant on the boundary condition is investigated.

We now follow Dai and Freed in [13] and generalize (8.1) and (8.2) to the parameterized context. As a general reference for the analysis of elliptic families we refer to the book [5].

As parameter space we take

$$
\mathscr{P}_{\Sigma}=\mathscr{F}_{\Sigma} \times \mathscr{M}_{\Sigma},
$$

where $\mathscr{M}_{\Sigma}$ denotes the space of Riemannian metrics on $\Sigma$ and $\mathscr{F}_{\Sigma}$ denotes the space of flat connections on the bundle $\left.E\right|_{\Sigma} \rightarrow \Sigma$.

Given a manifold $X$ with $\partial X=\Sigma$, we will also use the parameter space

$$
\mathscr{P}_{X}=\mathscr{F}_{X} \times \mathscr{M}_{\Sigma}
$$


where $\mathscr{F}_{X}$ denotes the space of flat connections on $E \rightarrow X$ in cylindrical form $B=b+\frac{\partial}{\partial x}$ on a collar. Notice that we take the space of Riemannian metrics on $\Sigma$, not $X$, to define $\mathscr{P}_{X}$.

Also note that in contrast to [13] the parameter spaces $\mathscr{P}_{\Sigma}$ and $\mathscr{P}_{X}$ are infinitedimensional. For the discussion of the determinant line bundle, however, this does not cause any additional difficulties. The reader who prefers not to worry about the manifold structure of $\mathscr{P}_{X}, \mathscr{P}_{\Sigma}$ may think of having chosen a finitedimensional submanifold of $\mathscr{P}_{X}, \mathscr{P}_{\Sigma}$.

To be more precise we consider the trivial fibration

$$
\pi: X \times \mathscr{P}_{X} \rightarrow \mathscr{P}_{X} .
$$

Give the fiber $X_{p}$ over $p=(B, g)$ a Riemannian metric so that in a fixed collar of $\partial X$ the metric takes the form $g+d u^{2}$. This can be done in a smooth way over $\mathscr{P}_{X}$; for example, fix a metric on the interior of $X$ and use a cutoff function in a slightly larger collar to interpolate between this fixed metric and $g+d u^{2}$. Call the resulting extended metric $\tilde{g}$. The fiber of the relative tangent bundle $T\left(X \times \mathscr{P}_{X} / \mathscr{P}_{X}\right):=\operatorname{ker} T \pi: T\left(X \times \mathscr{P}_{X}\right) \rightarrow T \mathscr{P}_{X}$ over the point $(x,(B, g)) \in$ $X \times \mathscr{P}_{X}$ is given by $T_{x} X \oplus 0$ and the metric on $T_{(x,(B, g))}\left(X \times \mathscr{P}_{X} / \mathscr{P}_{X}\right)$ is $\tilde{g}_{x}$.

Since $\pi$ is a product we have a natural horizontal structure which is given by the kernel of the tangent map of the projection $X \times \mathscr{P}_{X} \rightarrow X$ onto the first factor.

Summing up we have a Riemannian structure on the fibration $\pi$ in the sense of [13, p. 5159] resp. [5, Sec. 10.1].

Given $p=(b, g) \in \mathscr{P}_{\Sigma}$ we modify the previous notation slightly and denote by $A_{p}$ the odd signature operator coupled to $b$ in order to emphasize its dependence on the Riemannian metric $g$. Then $A_{p}$ acts on the bundle $(\Lambda \mathscr{T})_{p}:=\Lambda T^{*}(\Sigma ; E):=\oplus_{q \geq 0} \Lambda^{q} T^{*}(\Sigma ; E)$, where the notation $(\Lambda \mathscr{T})_{p}$ also emphasizes the dependence on $p$ through the metric. In fact $(\Lambda \mathscr{T})_{p}$ is the restriction of the bundle $\Lambda \mathscr{T}:=\Lambda T^{*}\left(\Sigma \times \mathscr{P}_{\Sigma} / \mathscr{P}_{\Sigma}, E\right):=\oplus_{q \geq 0} \Lambda^{q} T^{*}\left(\Sigma \times \mathscr{P}_{\Sigma} / \mathscr{P}_{\Sigma}, E\right)$ (the exterior power bundle of the relative cotangent bundle) to the fiber $\Sigma \times\{p\}$. Thus, the $\left(A_{p}\right)_{p \in \mathscr{P}_{\Sigma}}$ form a smooth family of Dirac type operators in the sense of $[5$, Sec. 9.2].

We then form the associated determinant bundle $\operatorname{det} \operatorname{ker} A \rightarrow \mathscr{P}_{\Sigma}$ whose fiber over $p \in \mathscr{P}_{\Sigma}$ is $\operatorname{det} \operatorname{ker} A_{p}=\left(\operatorname{det} \operatorname{ker} A_{p}^{+}\right)^{-1} \otimes \operatorname{det} \operatorname{ker} A_{p}^{-}$. One can find a careful construction of $\operatorname{det} \operatorname{ker} A$ in many articles, e.g. [5, 6], as well as the construction of the Quillen metric and connection on $\operatorname{det} \operatorname{ker} A$. We outline briefly the reason why the fibers $\operatorname{det}$ ker $A_{p}$ glue together to form a smooth bundle for the benefit 
of the reader. The space $\mathscr{P}_{\Sigma}$ is covered by open sets $U_{\mu}, \mu>0$, consisting of those $p$ so that $\mu \notin \operatorname{Spec}\left(A_{p}\right)$. If $F_{p}^{ \pm}(\mu)$ denotes the span of those eigenvectors of $A_{p}$ whose eigenvalues lie in $(-\mu, \mu) \backslash\{0\}$, then the vector spaces $H(\mu)_{p}$ defined by

$$
H(\mu)_{p}:=F_{p}^{+}(\mu) \oplus \operatorname{ker} A_{p} \oplus F_{p}^{-}(\mu)
$$

form a smooth, finite dimensional vector bundle over $U_{\mu}$, whose fibers are invariant under $\gamma_{p}$, since $\gamma_{p} A_{p}=-A_{p} \gamma_{p}$. Thus $H(\mu)_{p}$ is a Hermitian symplectic space and so has a decomposition

$$
H(\mu)_{p}=\left(H^{+}(\mu)_{p} \oplus \operatorname{ker} A_{p}^{+}\right) \oplus\left(\operatorname{ker} A_{p}^{-} \oplus H^{-}(\mu)_{p}\right)
$$

into the $\pm i$ eigenspaces of $\gamma_{p}$ acting on $H(\mu)_{p}$. The decomposition (8.4) yields

$$
\operatorname{det} H(\mu)_{p}=\operatorname{det}\left(\operatorname{ker} A_{p}\right) \otimes \operatorname{det}\left(H^{+}(\mu)_{p}\right)^{-1} \otimes \operatorname{det}\left(H^{-}(\mu)_{p}\right) .
$$

The crucial observation now is that $\operatorname{det}\left(H^{+}(\mu)_{p}\right)^{-1} \otimes \operatorname{det}\left(H^{-}(\mu)_{p}\right)$ is canonically trivial since $\operatorname{det}\left(A_{p}^{+}: H^{+}(\mu)_{p} \rightarrow H^{-}(\mu)_{p}\right)$ is a canonical nonzero section of $\operatorname{det}\left(H^{+}(\mu)_{p}\right)^{-1} \otimes \operatorname{det}\left(H^{-}(\mu)_{p}\right)$. Consequently $\operatorname{det} H(\mu)_{p}$ is canonically isomorphic to $\operatorname{det} \operatorname{ker} A_{p}$. Identifying $\operatorname{det} \operatorname{ker} A \mid U_{\mu}$ with $\operatorname{det} H(\mu) \mid U_{\mu}$ shows that $\operatorname{det} \operatorname{ker} A$ indeed is a smooth line bundle over $\mathscr{P}_{\Sigma}$.

For future reference we denote the canonical (bundle) isomorphism

$$
\varphi(\mu): \operatorname{det}(\operatorname{ker} A)\left|U_{\mu} \longrightarrow \operatorname{det} H(\mu)\right| U_{\mu}, \quad \xi_{p} \mapsto \xi_{p} \otimes \operatorname{det}\left(A_{p}^{+} \mid H^{+}(\mu)\right) .
$$

From (8.1) one now infers (cf. [13]):

Proposition 8.1 Given $p=\left(b_{p}, g_{p}\right) \in \mathscr{P}_{\Sigma}$, let $A_{\theta, p}$ denote the odd signature operator on the trivial bundle $\mathbb{C}^{n} \times \Sigma \rightarrow \Sigma$ with respect to the Riemannian metric $g_{p}$ on $\Sigma$.

Then the vector spaces $\operatorname{det}\left(\operatorname{ker} A_{p}\right)^{-1} \otimes \operatorname{det}\left(\operatorname{ker} A_{\theta, p}\right)$ form a smooth vector bundle

$$
\operatorname{det}(\operatorname{ker} A)^{-1} \otimes \operatorname{det}\left(\operatorname{ker} A_{\theta}\right) \rightarrow \mathscr{P}_{\Sigma} .
$$

Moreover, if $\partial X=\Sigma$ the $\rho$-invariant defines a smooth lift

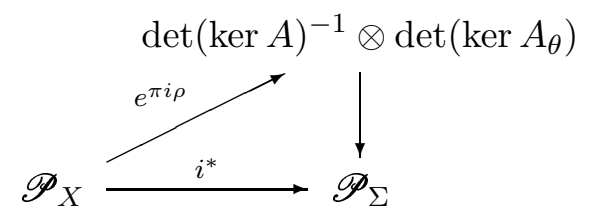

where $i^{*}$ denotes the restriction.

Algebraic 83 Geometric Topology, Volume 3 (2003) 
Proof From the previous discussion and in view of (8.1) the statement of the proposition is clear except the fact that $e^{\pi i \rho}$ defines a smooth lift into $\operatorname{det}(\operatorname{ker} A)^{-1} \otimes \operatorname{det}\left(\operatorname{ker} A_{\theta}\right)$. Although this fact was proved in [13] it also follows from our results. The key is that $(8.1)$ can be generalized in such a way that one obtains smooth sections over $U_{\mu}$. For a Lagrangian $W \subset H(\mu)_{p}$ denote by $\eta\left(D_{B, W}, X\right)$ the $\eta$-invariant of $D_{B}$ with respect to the boundary condition given by the orthogonal projection onto $\left(F_{p}^{+} \ominus F_{p}^{+}(\mu)\right) \oplus W$. Denote by $\phi(\mu)_{p}(W)$ the analogue of $\phi(W)$ for the space $H(\mu)_{p}$. Then $\operatorname{det}\left(\phi(\mu)_{p}(W)\right)$ is a canonical element of $\operatorname{det} H(\mu)_{p}$.

Given two such subspaces $W_{1}, W_{2}$ then by [26, Thm. 4.2$]$ we have the following generalization of (8.1):

$$
e^{2 \pi i \tilde{\eta}\left(D_{B, W_{1}}, X\right)} \operatorname{det}\left(\phi(\mu)_{p}\left(W_{1}\right)\right)^{-1}=e^{2 \pi i \tilde{\eta}\left(D_{B, W_{2}}, X\right)} \operatorname{det}\left(\phi(\mu)_{p}\left(W_{2}\right)\right)^{-1} .
$$

This shows that the expression $e^{2 \pi i \tilde{\eta}\left(D_{B, W_{1}}, X\right)} \operatorname{det}\left(\phi(\mu)_{p}\left(W_{1}\right)\right)^{-1}$ is independent of $W_{1}$ and choosing a smooth family of Lagrangians in $\left(H(\mu)_{p}\right)_{p \in U_{\mu}}$ over $U_{\mu}$ gives a smooth section of $\operatorname{det}(\operatorname{ker} A)$.

The (tensor product of two) determinant bundle(s)

$$
\operatorname{det}(\operatorname{ker} A)^{-1} \otimes \operatorname{det}\left(\operatorname{ker} A_{\theta}\right) \rightarrow \mathscr{P}_{\Sigma}
$$

admits the Quillen metric [32] and its natural compatible connection $\nabla^{Q}[6]$. The main result of [13] can be used to compute $\nabla^{Q}\left(e^{i \pi \rho}\right)$.

Let us first briefly recall the main facts about metrics and connections on $\operatorname{det}(\operatorname{ker} A)\left(\right.$ resp. $\left.\operatorname{det}(\operatorname{ker} A)^{-1} \otimes \operatorname{det}\left(\operatorname{ker} A_{\theta}\right)\right)$. We use the notation from page 661. Since the fiber of the relative tangent bundle $T\left(\Sigma \times \mathscr{P}_{\Sigma} / \mathscr{P}_{\Sigma}, E\right)$ over $(x, p) \in \Sigma \times \mathscr{P}_{\Sigma}$ is $T_{x} \Sigma \oplus 0$, the relative tangent bundle is naturally a Riemannian vector bundle. Consequently, $\Lambda \mathscr{T}$ inherits a natural metric from the relative tangent bundle. Furthermore, by [5, Prop. 10.2] $T\left(\Sigma \times \mathscr{P}_{\Sigma} / \mathscr{P}_{\Sigma}, E\right)$ has a natural connection which is induced solely by the Riemannian structure of the fibration $\pi$. This connection induces a connection on the bundle $\Lambda \mathscr{T}$. Finally, we note that for each $p$ the metric on $\Lambda \mathscr{T}$ induces an $L^{2}$-structure on sections of $(\Lambda \mathscr{T})_{p}$. In the terminology of family index theory the space $C^{\infty}\left(\Sigma \times\{p\}, \Lambda \mathscr{T}_{p}\right)$ of sections of $(\Lambda \mathscr{T})_{p}$ is viewed as the fiber over $p$ of an infinite-dimensional Hermitian bundle $\pi_{*} \Lambda \mathscr{T}$ whose sections are $C^{\infty}\left(\Sigma \times \mathscr{P}_{\Sigma}, \Lambda \mathscr{T}\right)$. By [5, Prop. 9.13] there is a natural connection $\nabla^{\pi_{*} \Lambda \mathscr{T}}$ on $\pi_{*} \Lambda \mathscr{T}$ which is compatible with the inner product. For details we refer to [5, Chap. 9].

The bundle $H(\mu)$ is a finite-dimensional sub-bundle of $\left(C^{\infty}\left(\Sigma \times\{p\}, \Lambda \mathscr{T}_{p}\right)\right)_{p \in U_{\mu}}$ and $\nabla^{\pi_{*} \Lambda \mathscr{T}}$ projects to a connection $\nabla^{\mu}$ on $H(\mu)$. Furthermore, $H(\mu)$ inherits a metric $h$ from the $L^{2}$-structure on $\left(C^{\infty}\left(\Sigma \times\{p\}, \Lambda \mathscr{T}_{p}\right)\right)_{p \in U_{\mu}}$. 
However, the bundle map $\varphi(\mu)(8.6)$ is not an isometry with respect to the metric $h$ and thus $h$ does in general not descend to a smooth metric on $\operatorname{det} \operatorname{ker} A \mid U_{\mu}$. The norm of $\varphi(\mu)$ (with respect to the natural metrics on $\operatorname{det}$ ker $A \mid U_{\mu}$ and on $\operatorname{det} H(\mu))$ is given by

$$
\begin{aligned}
\left\|\varphi(\mu)_{p}\right\| & =\left(\operatorname{det} A_{p}^{+} A_{p}^{-} \mid H^{+}(\mu)_{p}\right)^{1 / 2} \\
& =\prod_{\lambda \in \operatorname{Spec}\left(A_{p}\right), 0<\lambda<\mu} \lambda .
\end{aligned}
$$

Therefore, the Quillen metric defined by

$$
h^{Q}(\xi):=h(\xi) \prod_{\lambda \in \operatorname{Spec} A_{p}} \lambda:=h(\xi) \operatorname{det}_{\zeta}\left(A_{p}^{+} A_{p}^{-} \mid \operatorname{ker} A_{p}^{\perp}\right)
$$

for $\xi \in \operatorname{det} \operatorname{ker} A_{p}$ is a smooth metric on $\operatorname{det} \operatorname{ker} A$. Here $\operatorname{det}_{\zeta}\left(A_{p}^{+} A_{p}^{-} \mid \operatorname{ker} A_{p}^{\perp}\right)$ denotes the $\zeta$-regularized determinant of the operator $A_{p}^{+} A_{p}^{-} \mid \operatorname{ker} A_{p}^{\perp}$.

The natural connection $\nabla^{\operatorname{det}, \mu}$ on $\operatorname{det} \operatorname{ker} A \mid U_{\mu}$ induced by $\nabla^{\mu}$ is in general not compatible with the Quillen metric. However, there is a connection, $\nabla^{Q}$, on $\operatorname{det} \operatorname{ker} A$ which is compatible with the Quillen metric. Formally, one has over $U_{\mu}$

$$
\begin{aligned}
\nabla^{Q} & =\nabla^{\operatorname{det}, \mu}+\operatorname{tr}\left(\left(A^{+}\right)^{-1} \nabla^{\mu} A^{+}\right) \\
& =: \nabla^{\text {det }, \mu}+\beta_{+}^{\mu} .
\end{aligned}
$$

The right hand side of (8.8) has to be suitably regularized; for details we refer to $[5$, Sec. 9.7].

As explained on page 661 the fibration $X \times \mathscr{P}_{X}$ has naturally the structure of a Riemannian fibration in the sense of $[13$, p. 5159] resp. [5, Sec. 10.1]. Thus by [5, Prop. 10.2] the relative tangent bundle $T\left(X \times \mathscr{P}_{X} / \mathscr{P}_{X}\right)$ has a natural connection, $\nabla^{T\left(X \times \mathscr{P}_{X} / \mathscr{P}_{X}\right)}$. Let $R^{X} \in \Omega_{X \times \mathscr{P}_{X}}^{2}\left(\operatorname{End} T\left(X \times \mathscr{P}_{X} / \mathscr{P}_{X}\right)\right)$ be the curvature of this connection.

Next, let $\mathscr{E} \rightarrow X \times \mathscr{P}_{X}$ be the pullback of the bundle $E \rightarrow X$ via the projection $X \times \mathscr{P}_{X} \rightarrow X$. Let $\nabla^{\mathbf{B}}$ denote the connection on $\mathscr{E}$ whose restriction to each fiber $X_{B, g}$ is $B$. This can be constructed by choosing an arbitrary connection on $\mathscr{E}$ and then adjusting it by the appropriate 1 -form. The curvature of $\nabla^{\mathbf{B}}$, $F^{\mathbf{B}} \in \Omega_{X \times \mathscr{P}_{X}}^{2}($ End $\mathscr{E})$ restricts to zero in each fiber $X_{B, g}$ since $B$ is flat.

Similarly we construct the trivial connection $\nabla^{\Theta}$ by replacing $\mathscr{E}$ by the trivial bundle and $B$ by the trivial connection in the above formula. Its curvature $F^{\Theta}$ is zero and so the Chern character $\operatorname{ch}\left(F^{\boldsymbol{\Theta}}\right)=n$ (as a form).

Then with these preparations, the Dai-Freed theorem implies the following. 


\section{Proposition 8.2}

$$
\nabla^{Q}\left(e^{i \pi \rho}\right)=2^{(\operatorname{dim} X-1) / 2}\left(\int_{X} L\left(R^{X}\right)\left(\operatorname{ch}\left(F^{\mathbf{B}}\right)-\operatorname{ch}\left(F^{\boldsymbol{\Theta}}\right)\right)\right)_{[1]} \cdot e^{i \pi \rho},
$$

where $\int_{X}$ denotes integration over the fibers and the subscript "[1]" means the 1-form part of an inhomogeneous differential form. Furthermore,

$$
L\left(R^{X}\right)=\operatorname{det}^{1 / 2}\left(\frac{R^{X} / 2}{\tanh \left(R^{X} / 2\right)}\right)
$$

denotes the Hirzebruch $L$-form associated to $R^{X}$.

Proof In [13] the theorem was stated for a smooth family of spin Dirac operators. However, as they pointed out, their result remains true for twisted Dirac operators. Since the integrand in the right hand side of (8.9) is local and since every manifold is locally spin let us assume for the moment that $X$ is spin. The complex Clifford algebra $\mathbb{C} l_{2 k+1}$ (remember $\operatorname{dim} X=2 k+1$ ) has two inequivalent irreducible respresentations $\Delta^{ \pm}$and hence

$$
\mathbb{C} l_{2 k+1} \cong \operatorname{End}\left(\Delta^{+}\right) \oplus \operatorname{End}\left(\Delta^{-}\right) .
$$

Denote by $S_{\mathbb{C}}^{ \pm} \rightarrow X \times \mathscr{P}_{X}$ the spinor bundles corresponding to $\Delta^{ \pm}$associated to the relative tangent bundle $T\left(X \times \mathscr{P}_{X} / \mathscr{P}_{X}\right)$. These inherit natural connections, $\nabla^{ \pm}$, from the connection $\nabla^{T\left(X \times \mathscr{P}_{X} / \mathscr{P}_{X}\right)}$ on the relative tangent bundle $T\left(X \times \mathscr{P}_{X} / \mathscr{P}_{X}\right)$.

From the decomposition (8.10) one easily infers (cf. also [5, Sec. 4.1] for the even dimensional case) that the odd signature operator $D_{\Theta}$ is the spin Dirac operator coupled to the twisting bundle $\left(S_{\mathbb{C}}^{+} \otimes \mathbb{C}^{n}, \nabla^{+} \otimes \mathrm{id}+\mathrm{id} \otimes \nabla^{\Theta}\right)$. Analogously, $D_{B}$ is the spin Dirac operator coupled to the twisting bundle $\left(S_{\mathbb{C}}^{+} \otimes E, \nabla^{+} \otimes\right.$ $\mathrm{id}+\mathrm{id} \otimes \nabla^{B}$ ). Consequently, [13, Theorem 1.9] yields

$$
\nabla^{Q}\left(e^{i \pi \rho}\right)=\left(\int_{X} \hat{A}\left(R^{X}\right) \wedge \operatorname{ch}\left(S_{\mathbb{C}}^{+}, \nabla^{+}\right) \wedge\left(\operatorname{ch}\left(F^{\mathbf{B}}\right)-\operatorname{ch}\left(F^{\Theta}\right)\right)\right)_{[1]} \cdot e^{i \pi \rho} .
$$

It remains to identify the differential form $\hat{A}\left(R^{X}\right) \wedge \operatorname{ch}\left(S_{\mathbb{C}}^{+}, \nabla^{+}\right)$. By the following Lemma we have

$$
\hat{A}\left(R^{X}\right) \wedge \operatorname{ch}\left(S_{\mathbb{C}}^{+}, \nabla^{+}\right)=2^{k} L\left(R^{X}\right)=2^{k} \operatorname{det}^{1 / 2}\left(\frac{R^{X} / 2}{\tanh \left(R^{X} / 2\right)}\right) .
$$

Note that although (8.12) is an identity between differential forms, it is in fact a statement about invariant polynomials on the special orthogonal group and hence it follows indeed from the next lemma. 
We point out that it is crucial in the following that $\hat{A}\left(R^{X}\right) \wedge \operatorname{ch}\left(S_{\mathbb{C}}^{+}, \nabla^{+}\right)=$ $2^{k} L\left(R^{X}\right)$ is a Pontrjagin form and hence is a sum of differential forms of degree divisible by four.

Lemma 8.3 Let $M$ be a differentiable manifold and $E \rightarrow M$ a real oriented vector bundle of rank $2 k+1$ which carries a spin structure. Let $S_{\mathbb{C}}^{ \pm}(E)$ be the corresponding spinor bundles. Then $\hat{A}(E) \wedge \operatorname{ch}\left(S_{\mathbb{C}}^{ \pm}(E)\right)=2^{k} L(E)$.

Proof A similar result for even rank bundles is well-known (the lemma is probably well-known also, however standard texts refer to the even dimensional case only; see [5, Sec. 4.1], [16, Sec. 3.3.5], [28, Sec. III.11]) and we will reduce the lemma to the even dimensional case. By the splitting principle and since the bundle is orientable we may assume that

$$
E \simeq \tilde{E} \oplus \mathbb{R}_{M},
$$

where $\tilde{E}$ is a real oriented bundle of rank $2 k$ and $\mathbb{R}_{M}$ denotes the trivial $\mathbb{R}$ bundle over $M$ (cf. [28, Rem. III.11.3]). Denote by $S_{\mathbb{C}}(\tilde{E})$ the unique complex spinor bundle associated to the spin structure on $\tilde{E}$. Then the representation theory of the complex Clifford algebras immediately implies that

$$
S_{\mathbb{C}}^{+}(E) \simeq S_{\mathbb{C}}^{-}(E) \simeq S_{\mathbb{C}}(\tilde{E})
$$

(isomorphisms as complex vector bundles). Hence we are reduced to the even rank case and it follows (see the references above)

$$
\hat{A}(E) \wedge \operatorname{ch}\left(S_{\mathbb{C}}^{ \pm}(E)\right)=\hat{A}(\tilde{E}) \wedge \operatorname{ch}\left(S_{\mathbb{C}}(\tilde{E})\right)=2^{k} L(\tilde{E})=2^{k} L(E) .
$$

Proposition 8.2 implies the following.

Theorem 8.4 If one restricts to $S U(n)$ connections on $E \rightarrow X$ or if $X$ is $(4 \ell-1)$-dimensional then

$$
\nabla^{Q}\left(e^{\pi i \rho}\right)=0
$$

Proof Recall that $X$ is a $(2 k-1)$-dimensional manifold. Decompose the differential form $\operatorname{ch}\left(F^{\mathbf{B}}\right)-\operatorname{ch}\left(F^{\boldsymbol{\Theta}}\right)$ into its homogeneous components:

$$
\operatorname{ch}\left(F^{\mathbf{B}}\right)-\operatorname{ch}\left(F^{\Theta}\right)=\operatorname{ch}_{2}\left(F^{\mathbf{B}}\right)+\operatorname{ch}_{4}\left(F^{\mathbf{B}}\right)+\operatorname{ch}_{6}\left(F^{\mathbf{B}}\right)+\cdots
$$

Similarly decompose

$$
L\left(R^{X}\right)=L_{0}\left(R^{X}\right)+L_{4}\left(R^{X}\right)+L_{8}\left(R^{X}\right)+\cdots
$$


Thus

$$
\left(\int_{X} L\left(R^{X}\right)\left(\operatorname{ch}\left(F^{\mathbf{B}}\right)-\operatorname{ch}\left(F^{\mathbf{\Theta}}\right)\right)\right)_{[1]}=\sum_{q \geq 1} \int_{X} L_{2 k-2 q}\left(R^{X}\right) \operatorname{ch}_{2 q}\left(F^{\mathbf{B}}\right) .
$$

Since the restriction of $F^{\mathbf{B}}$ to $X_{p}$ is flat, $\operatorname{ch}_{2 q}\left(F^{\mathbf{B}}\right)=\operatorname{const}(q) \operatorname{Tr}\left(\left(F^{\mathbf{B}}\right)^{q}\right)$ has at most $q$ components in the " $X$ " direction, i.e. writing $\operatorname{ch}_{2 q}\left(F^{\mathbf{B}}\right)$ locally as a sum

$$
\operatorname{ch}_{2 q}\left(F^{\mathbf{B}}\right)_{x, p}=\sum_{i=0}^{2 q} f_{x, p}^{i} \alpha_{i} \wedge \beta_{2 q-i}
$$

with $\alpha_{i} \in \Omega_{X}^{i}$ and $\beta_{2 q-i} \in \Omega_{\mathscr{P}_{X}}^{2 q-i}$, then $f^{i}=0$ for $i>q$.

This implies that the only possible non-zero summand in the right side of (8.15) is the term with $q=1$, i.e.

$$
\int_{X} L_{2 k-2}\left(R^{X}\right) \wedge \operatorname{ch}_{2}\left(F^{\mathbf{B}}\right)
$$

But since $\operatorname{ch}_{2}\left(F^{\mathbf{B}}\right)=c_{1}\left(F^{\mathbf{B}}\right)$ and $L_{2 k-2}\left(R^{X}\right)=0$ if $2 k-2$ is not divisible by $4,(8.16)$ vanishes if $\mathbf{B}$ is an $S U(n)$ connection or if $2 k-2 \neq 4 \ell$. The result now follows from Proposition 8.2.

The following theorem exhibits a functoriality property of $\rho$ modulo $\mathbb{Z}$ for manifolds with boundary. It is closely related to Theorem 7.2 , but the weaker hypothesis ( $F$ need not be a homotopy equivalence) gives a weaker conclusion: the $\rho$-invariants agree only modulo $\mathbb{Z}$.

Theorem 8.5 Let $X$ and $X^{\prime}$ be two odd dimensional manifolds and suppose that $F: X^{\prime} \rightarrow X$ is a smooth map such that the restriction $f=\left.F\right|_{\partial X^{\prime}}: \partial X^{\prime} \rightarrow$ $\partial X$ is a diffeomorphism. Let $\alpha_{0}, \alpha_{1}: \pi_{1}(X) \rightarrow S U(n)$ be two representations in the same path component of $\chi\left(\pi_{1}(X), S U(n)\right)$. Let $g_{0}$ and $g_{1}$ be two metrics on $\partial X$. Then

$$
\begin{aligned}
& \rho\left(X, \alpha_{1}, g_{1}\right)-\rho\left(X, \alpha_{0}, g_{0}\right) \\
& \quad \equiv \rho\left(X^{\prime}, F^{*}\left(\alpha_{1}\right), f^{*}\left(g_{1}\right)\right)-\rho\left(X^{\prime}, F^{*}\left(\alpha_{0}\right), f^{*}\left(g_{0}\right)\right) \quad(\bmod \mathbb{Z}) .
\end{aligned}
$$

In particular, if $F: X^{\prime} \rightarrow X$ induces an isomorphism on fundamental groups then there is a factorization

$$
\begin{array}{r}
\chi\left(\pi_{1}(X), S U(n)\right) \times \mathscr{M}_{\Sigma} / \mathscr{D}_{\Sigma}^{0} \underset{. .}{\stackrel{\rho(X)-\rho\left(X^{\prime}\right)}{\longrightarrow}} \mathbb{R} / \mathbb{Z} \\
\pi_{0}\left(\chi\left(\pi_{1}(X), S U(n)\right)\right) \times \mathscr{M}_{\Sigma} / \mathscr{D}_{\Sigma}^{0}
\end{array}
$$

and $\rho(X)-\rho\left(X^{\prime}\right)$ is zero on the path component of the trivial representation. The result holds for $U(n)$ replacing $S U(n)$ if $\operatorname{dim} X=4 \ell-1$. 
Proof The map $F: X^{\prime} \rightarrow X$ induces a map $F^{*}$ on flat connections $\mathscr{F}_{X} \rightarrow$ $\mathscr{F}_{X^{\prime}}$ by pulling back connections. Using $f$ to pull back metrics on the boundary we see that $F$ induces a map $F^{*}: \mathscr{P}_{X} \rightarrow \mathscr{P}_{X^{\prime}}$ so that

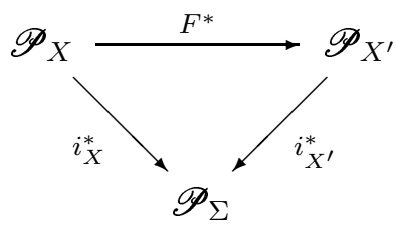

commutes.

Let $\alpha_{t}$ be a path of representations from $\alpha_{0}$ to $\alpha_{1}$. Such a path can be chosen to be piecewise analytic and a corresponding piecewise analytic path of flat connections $B_{t}$ with holonomy $\alpha_{t}$ can be found ([15]). By adding the results in the end we may assume that the path $B_{t}$ is analytic, and hence smooth. Let $g_{t}$ be a smooth path of metrics on $\partial X$ from $g_{0}$ to $g_{1}$. We identify $\partial X$ and $\partial X^{\prime}$ via $f$.

Choose a Lagrangian subspace $V_{t}$ in $\operatorname{ker} A_{\left(b_{t}, g_{t}\right)}$ for each $t$ so that $V_{0}=V_{X, \alpha_{0}}$ and $V_{1}=V_{X, \alpha_{1}}$. (See Lemma 7.3.) Similarly choose a Lagrangian subspace $W_{t}$ of $\operatorname{ker} A_{\left(\theta, g_{t}\right)}$ with $W_{t}=V_{X, \tau}$.

Let $c$ be the real number $c=\rho\left(X, \alpha_{0}, g_{0}\right)-\rho\left(X^{\prime}, \alpha_{0}, g_{0}\right)$. Notice that by definition $c=0$ if $\alpha_{0}$ is trivial. The smooth sections

$$
\phi_{1}: t \mapsto \exp \left(\pi i\left(\rho\left(X, \alpha_{t}, V_{t}, W_{t}\right), g_{t}\right)\right) \operatorname{det}\left(\phi\left(V_{t}\right)\right) \operatorname{det}\left(\phi\left(W_{t}\right)\right)^{-1}
$$

and

$$
\phi_{2}: t \mapsto e^{c \pi i} \exp \left(\pi i\left(\rho\left(X^{\prime}, \alpha_{t}, V_{t}\right), g_{t}\right)\right) \operatorname{det}\left(\phi\left(V_{t}\right)\right) \operatorname{det}\left(\phi\left(W_{t}\right)\right)^{-1}
$$

agree at $t=0$ and by Theorem 8.4 satisfy $\nabla^{Q}\left(\phi_{1}\right)=0=\nabla^{Q}\left(\phi_{2}\right)$ (since $e^{c \pi i}$ is constant). In other words, $\phi_{1}$ and $\phi_{2}$ are two horizontal lifts of the path $[0,1] \rightarrow \mathscr{P}_{\Sigma}, t \mapsto\left(b_{t}, g\right)$. Since they agree at $t=0$, they agree for all $t$. In particular, at $t=1$ we conclude

$$
e^{\pi i \rho\left(X, \alpha_{1}, g_{1}\right)}=e^{\pi i \rho\left(X^{\prime}, \alpha_{1}, g_{1}\right)} e^{c \pi i} .
$$

This proves the first part of the theorem. The second part follows from the first and the discussion following Theorem 5.1.

The second statement in Theorem 8.5 should be compared to [14, Theorem 7.1].

We end this section with a discussion which shows that finding an explicit dependence of the $\rho$-invariant on the metric on the boundary is ultimately tied to the delicate construction of the connection $\nabla^{Q}$. 
Suppose that a representation $\alpha: \pi_{1} X \rightarrow U(n)$ is fixed and consider the function of metrics on the boundary

$$
\rho(X, \alpha): \mathscr{M}_{\Sigma} \rightarrow \mathbb{R}
$$

This function is smooth, since $e^{i \pi \rho}$ is smooth and since the dimension of the kernel of $D_{B, V_{X, \alpha}}$ is independent of the metric by (2.3).

Proposition 8.6 With the denotations of (8.8) we have

$$
\begin{aligned}
d \rho(X, \alpha) & =-\frac{1}{\pi i} \frac{\operatorname{det} \phi\left(V_{X, \alpha}\right)}{\operatorname{det} \phi\left(V_{X, \tau}\right)} \nabla^{Q} \frac{\operatorname{det} \phi\left(V_{X, \tau}\right)}{\operatorname{det} \phi\left(V_{X, \alpha}\right)} \\
& =-\frac{1}{\pi i} \frac{\operatorname{det} \phi\left(V_{X, \alpha}\right)}{\operatorname{det} \phi\left(V_{X, \tau}\right)} \nabla \frac{\operatorname{det} \phi\left(V_{X, \tau}\right)}{\operatorname{det} \phi\left(V_{X, \alpha}\right)}-\frac{1}{\pi i} \beta_{+} .
\end{aligned}
$$

Proof For the purpose of this proof the abusive notation $e^{i \pi \rho}$ introduced at the beginning of this section is too confusing. During this proof we write $s(X, \alpha, g) s(X, \tau, g)^{-1}$ for the element of $\left(\operatorname{det} \text { ker } A_{b}\right)^{-1} \otimes \operatorname{det} \operatorname{ker} A_{\theta}$ defined by (8.2) and $e^{\pi i \rho}$ denotes the number obtained by exponentiating the $\rho$-invariant.

We cannot apply Theorem 8.4 directly. However, since the parameter space is $\mathscr{M}_{\Sigma}$ and $\alpha$ is fixed we have $\operatorname{ch}\left(F^{\mathbf{B}}\right)-\operatorname{ch}\left(F^{\boldsymbol{\Theta}}\right)=0$ and hence by Proposition 8.2 and $(8.15)$

$$
\nabla^{Q}\left(s(X, \alpha, g) s(X, \tau, g)^{-1}\right)=0 .
$$

Over $\mathscr{M}_{\Sigma}$ we have

$$
s(X, \alpha, g) s(X, \tau, g)^{-1}=e^{\pi i \rho(X, \alpha, g)} \operatorname{det} \phi\left(V_{X, \alpha}\right)^{-1} \operatorname{det} \phi\left(V_{X, \tau}\right) .
$$

Consequently, (8.18) implies

$$
\begin{aligned}
& d \rho(X, \alpha, g) \\
& =-\frac{1}{\pi i} \operatorname{det} \phi\left(V_{X, \alpha}\right) \operatorname{det} \phi\left(V_{X, \tau}\right)^{-1} \nabla^{Q}\left(\operatorname{det} \phi\left(V_{X, \alpha}\right)^{-1} \operatorname{det} \phi\left(V_{X, \tau}\right)\right) \\
& =-\frac{1}{\pi i} \operatorname{det} \phi\left(V_{X, \alpha}\right) \operatorname{det} \phi\left(V_{X, \tau}\right)^{-1} \nabla\left(\operatorname{det} \phi\left(V_{X, \alpha}\right)^{-1} \operatorname{det} \phi\left(V_{X, \tau}\right)\right)-\frac{1}{\pi i} \beta_{+} .
\end{aligned}
$$

Looking at the definition of $\beta_{+}$we see that this result gives a link between the dependence of $\rho$ on the metric and the variation of the (regularized) determinant of the operator $A_{b}$. This perhaps explains why we cannot expect the $\rho$-invariant to be independent of the metric on the boundary. 


\section{Topological consequences}

It is known that the $\rho$-invariants distinguish homotopy equivalent lens spaces [37]. By contrast, Neumann showed in [31] that $\rho$ is a homotopy invariant for manifolds with free abelian fundamental groups.

This leaves the problem of deciding exactly to what extent the $\rho$-invariant is a homotopy invariant open. One interesting aspect of this problem is that it can be studied one fundamental group at a time.

A conjecture of Weinberger states (see [38]):

Conjecture A (Weinberger) If $M$ is a closed (2k-1)-manifold with torsionfree fundamental group then $\rho(M, \alpha)$ depends only on the homotopy type of $M$.

Thus Neumann showed that Conjecture A holds for free abelian groups. The Farber-Levine-Weinberger theorem solves the problem for those groups whose $U(n)$ character varieties are connected, such as free groups. Wall's calculations for lens spaces shows that the extension of the conjecture to all groups is false: cyclic groups provide examples.

We make the following extension of the conjecture of Weinberger.

Conjecture B Suppose that $F: X \rightarrow X^{\prime}$ is a homotopy equivalence of manifolds with torsion free fundamental groups which restricts to a diffeomorphism on the boundary. Endow the boundaries with Riemannian metrics $g, g^{\prime}$ so that the restriction to the boundary is an isometry.

Then for any any unitary representation $\alpha: \pi_{1} X \rightarrow U(n)$

$$
\rho(X, \alpha, g)=\rho\left(X^{\prime}, \alpha, g^{\prime}\right) .
$$

This implies Weinberger's conjecture. In this instance our Theorem 7.2 implies Conjecture B for those manifolds whose $U(n)$ character varieties are connected.

This reveals the following strategy for attacking Conjecture A.

Definition 9.1 We say a homotopy equivalence $F: M^{\prime} \rightarrow M$ between closed manifolds can be split along a separating hypersurface $\Sigma \subset M$ if, after a homotopy of $F$,

(1) $\quad F$ is smooth and transverse to $\Sigma$ and the restriction $F: F^{-1}(\Sigma) \rightarrow \Sigma$ is a diffeomorphism, and 
(2) writing $M=X \cup_{\Sigma} Y$ and $M^{\prime}=X^{\prime} \cup_{\Sigma} Y^{\prime}, F$ restricts to homotopy equivalences $X^{\prime} \rightarrow X$ and $Y^{\prime} \rightarrow Y$.

The problem of determining when a homotopy equivalence can be split along a hypersurface has been extensively studied; see e.g. [37, Chapter 12A] or [12].

We have the following result.

Theorem 9.2 Let $F: M \rightarrow M^{\prime}$ be a homotopy equivalence between closed manifolds which can be split along a hypersurface $\Sigma \subset M$. Write $M=X \cup_{\Sigma}$ $Y$. Suppose that $\alpha: \pi_{1} M \rightarrow U(n)$ is a unitary representation such that the restriction $\left.\alpha\right|_{X}$ (resp. $\left.\alpha\right|_{Y}$ ) lies in the path component of the trivial $U(n)$ representation of $\pi_{1} X$ (resp. $\left.\pi_{1} Y\right)$. Then $\rho(M, \alpha)=\rho\left(M^{\prime}, \alpha\right)$.

In particular, if the image of the restriction map $\chi\left(\pi_{1} M, U(n)\right) \rightarrow \chi\left(\pi_{1} X, U(n)\right)$ lies in a path component of $\chi\left(\pi_{1} X, U(n)\right)$ and similarly for $Y$ (this holds e.g. when $\chi\left(\pi_{1} X, U(n)\right)$ and $\chi\left(\pi_{1} Y, U(n)\right)$ are path connected $)$, then $\rho(M, \alpha)=$ $\rho\left(M^{\prime}, \alpha\right)$ for all $\alpha \in \chi\left(\pi_{1} M, U(n)\right)$.

Proof This follows by combining Theorems 3.2 and 7.2.

Notice that it is much more likely that the restrictions $\left.\alpha\right|_{X}$ and $\left.\alpha\right|_{Y}$ lie in the path component of the trivial connection than that $\alpha$ itself does, since $\pi_{1} M$ is the free product of $\pi_{1} X$ and $\pi_{1} Y$ amalgamated over $\pi_{1} \Sigma$. Hence in trying to deform $\left.\alpha\right|_{X}$ and $\left.\alpha\right|_{Y}$ in their representation spaces one is no longer constrained by the relations imposed by amalgamating over $\pi_{1} \Sigma$.

As an application, if $X$ and $Y$ are manifolds with boundary which have path connected character varieties, and $f, g: \partial X \rightarrow \partial Y$ are homotopic diffeomorphisms, then $X \cup_{f} Y$ and $X \cup_{g} Y$ are homotopy equivalent and $\rho\left(X \cup_{f} Y, \alpha\right)=$ $\rho\left(X \cup_{g} Y, \alpha\right)$ for all $U(n)$ representations $\alpha$. One can construct such examples so that $X \cup_{f} Y$ and $X \cup_{g} Y$ are not diffeomorphic.

The problem of determining the number of path components of $\chi(\pi, U(n))$ is tricky. Some examples of groups with $\chi(\pi, U(n))$ path connected include $\pi$ free or free abelian. An interesting family of torsion-free groups with path connected unitary representation spaces are the 2-generator groups $\left\langle x, y, \mid x^{p}=y^{q}\right\rangle$ for $p, q$ relatively prime. For a taste of the problem for some 3-manifold groups $\pi$ the reader might glance at [27] and [20]. Notice that the isomorphism class of the bundle $E \rightarrow X$ is fixed on any path component of $\chi\left(\pi_{1} X, U(n)\right)$, and so if $X$ admits non-isomorphic flat bundles (e.g. if $H^{2}(X ; \mathbb{Z})$ contains non-trivial torsion) then $\chi\left(\pi_{1} X, U(n)\right)$ cannot be path connected. 
Conjecture B does not hold without the requirement that the homotopy equivalence behave nicely on the boundary; this is exhibited by the example at the end of Section 7. We will explore examples and applications of Theorem 9.2 in a later article.

We end this article with speculation concerning the similarity between the constructions of Section 8 and the approach to studying TQFTs advocated in Atiyah's book [1].

Take $\Sigma$ to be a 2-manifold and restrict to Riemannian metrics on $\Sigma$ which have constant curvature 1,0 , or -1 . Then $\mathscr{M}_{\Sigma} / \mathscr{D}_{\Sigma}^{0}=\mathscr{T}_{\Sigma}$ is the Teichmüller space of $\Sigma$. Thus Theorem 8.4 defines a complex line bundle with connection over $\chi\left(\pi_{1} \Sigma, U(n)\right) \times \mathscr{T}_{\Sigma}$, and given any 3 -manifold $X$ with boundary $\Sigma$ one obtains from $\rho(X, \alpha, g)$ a horizontal cross section:

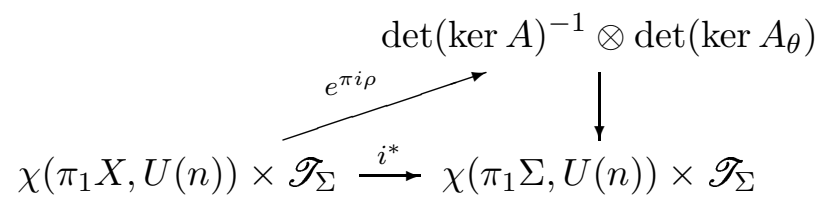

In [1] a similar diagram is obtained: a determinant bundle over $\chi\left(\pi_{1} \Sigma, U(n)\right)$ is constructed as follows. A metric $g \in \mathscr{T}_{\Sigma}$ defines a holomorphic structure on $\Sigma$. This defines a complex structure on $\chi\left(\pi_{1} \Sigma, U(n)\right)$ by identifying it with the moduli space of semi-stable holomorphic bundles over $\Sigma$. Then one takes the determinant bundle $D \rightarrow \chi\left(\pi_{1} \Sigma, U(n)\right)$ whose fiber over a point corresponding to a holomorphic bundle is the determinant of the corresponding $\bar{\partial}$-operator. The Quillen metric and the holomorphic structure on this determinant bundle determines a connection [32] which coincides with $\nabla^{Q}[6]$. Viewing holomorphic sections of this bundle as the fiber of a vector bundle over $\mathscr{T}_{\Sigma}$ defines a bundle which was shown to admit a projectively flat connection.

Defining a horizontal cross-section of $D$

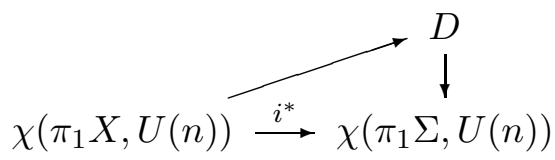

for a 3-manifold $X$ with boundary $\Sigma$ is problematic from this point of view. An alternative set-up is described in [33]. In that article a complex line bundle $L \rightarrow \chi\left(\pi_{1} \Sigma, S U(2)\right)$ is constructed using the Chern-Simons invariant $c s$. From 
the construction one immediately obtains the cross section

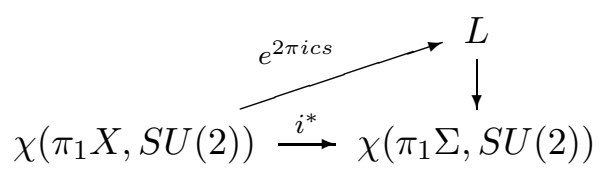

for any 3-manifold $X$ with boundary $\Sigma$ (this is a formalization of the fact that on a manifold with boundary, the Chern-Simons invariant is not a $U(1)=\mathbb{R} / \mathbb{Z}$ valued function but rather a cross section of a $U(1)$ bundle over the moduli space of the boundary). This part of the construction is independent of the choice of Riemannian metric on $\Sigma$. However, endowing $\Sigma$ with a metric defines a connection on $L$ for which the cross section is horizontal. It is shown in [33] that the line bundles $L$ and $D$ are isomorphic, linking the two approaches.

On a closed 3-manifold, the $S U(n)$ Chern-Simons invariants and $\rho$-invariants agree modulo $\mathbb{Z}$. This suggests that the set-up described in $[1,33]$ is related to our approach (encapsulated in the diagram (9.1)). It would be an interesting project to establish a precise relationship. Several complications arise. First, the $\rho$-invariant depends on the metric on $\Sigma$, as we have established, but the Chern-Simons invariant (viewed as a cross section of $L$ ) of a manifold with boundary is metric independent. Second, it is not clear how to relate the $\rho$ and Chern-Simons invariants for a manifold with boundary. In the closed case the Atiyah-Patodi-Singer index theorem provides the relationship, but generalizing this argument would require an extension of the Atiyah-Patodi-Singer theorem to manifolds with corners; a problem of significant interest that so far does not have a complete solution. Another interesting point is that the $\rho$-invariant is well-defined in $\mathbb{R}$, not just $\mathbb{R} / \mathbb{Z}$ as for the Chern-Simons invariant, and the cut-and-paste formula (2.6) holds in $\mathbb{R}$. This may give a clue as to how to refine the approach of [1]. Finally, since the set-up described in the present article works in any odd dimension, it may provide a direction to the problem of constructing some TQFTs in higher dimensions.

\section{References}

[1] M. F. Atryah, The geometry and physics of knots. Lezioni Lincee. [Lincei Lectures] Cambridge University Press, Cambridge, 1990.

[2] M. F. Atiyah, V. K. Patodi, and I. M. Singer, Spectral asymmetry and Riemannian geometry. I. Math. Proc. Cambridge Philos. Soc. 77 (1975), 43-69.

[3] M. F. Atiyah, V. K. Patodi, and I. M. Singer, Spectral asymmetry and Riemannian geometry. II. Math. Proc. Cambridge Philos. Soc. 78 (1975), no. 3, 405-432. 
[4] M. F. Atiyah, V. K. Patodi, and I. M. Singer, Spectral asymmetry and Riemannian geometry. III. Math. Proc. Cambridge Philos. Soc. 79 (1976), no. $1,71-99$.

[5] N. Berline, E. Getzler, and M. Vergne, Heat kernels and Dirac operators. Grundlehren der Mathematischen Wissenschaften, 298. Springer-Verlag, Berlin, 1992.

[6] J. M. Bismut and D. S. Freed, The analysis of elliptic families. I. Metrics and connections on determinant bundles. Comm. Math. Phys. 106 (1986), no. $1,159-176$.

[7] H. Boden, C. Herald, P. Kirk, and E. Klassen, Gauge theoretic invariants of Dehn surgeries on knots. Geom. Topol. 5 (2001), 143-226.

[8] B. Booss-Bavnbek, M. Lesch, and J. Phillips, Unbounded Fredholm operators and spectral flow. To appear in Canadian Journal of Mathematics, Math.FA/0108014.

[9] B. Booss-Bavnbek and K. P. Wojciechowski, Elliptic boundary problems for Dirac operators. Birkhäuser, Basel, 1993.

[10] U. Bunke, On the gluing problem for the $\eta$-invariant, J. Diff. Geom., Vol 41 (1995), 397-448

[11] S. Cappell, R. Lee, and E. Miller, On the Maslov index. Comm. Pure Appl. Math. 47 (1994), no. 2, 121-186.

[12] S. Cappell, A splitting theorem for manifolds. Invent. Math. 33 (1976), no. 2, 69-170.

[13] X. DAI and D. S. FreED, $\eta$-invariants and determinant lines. Topology and physics. J. Math. Phys. 35 (1994), no. 10, 5155-5194.

[14] M. S. FARBer and J. Levine, Jumps of the eta-invariant. With an appendix by Shmuel Weinberger: Rationality of $\rho$-invariants. Math. Z. 223 (1996), no. $2,197-246$.

[15] B. Fine, P. Kirk, and E. Klassen, A local analytic splitting of the holonomy map on flat connections. Math. Ann. 299 (1994), no. 1, 171-189.

[16] P.B. Gilkey, Invariance theory, the heat equation and the Atiyah-Singer index theorem. Second Edition, CRC Press, Boca Raton, 1995.

[17] J. Hempel, 3-Manifolds. Ann. of Math. Studies, No. 86. Princeton University Press, Princeton, N. J.; University of Tokyo Press, Tokyo, 1976.

[18] T. Kato, Perturbation theory for linear operators. Second edition. Grundlehren der Mathematischen Wissenschaften, Band 132. Springer-Verlag, Berlin-New York, 1976.

[19] P. KIRK and E. KLASSEN, Chern-Simons invariants of 3-manifolds and representation spaces of knot groups. Math. Ann. 287 (1990), no. 2, 343-367.

[20] P. KIRK and E. Klassen, Representation spaces of Seifert fibered homology spheres. Topology 30 (1991), no. 1, 77-95. 
[21] P. Kirk, E. Klassen and D. Ruberman, Splitting the spectral flow and the Alexander matrix. Comment. Math. Helv. 69 (1994), no. 3, 375-416.

[22] P. Kirk and E. KLAssen, Analytic deformations of the spectrum of a family of Dirac operators on an odd-dimensional manifold with boundary. Mem. Amer. Math. Soc. 124 (1996), no. 592.

[23] P. KiRK and E. KLASSEn, The spectral flow of the odd signature operator and higher Massey products. Math. Proc. Cambridge Philos. Soc. 121 (1997), no. 2, 297-320.

[24] P. KIRK and E. Klassen, The first-order spectral flow of the odd signature operator on a manifold with boundary. Topology Appl. 116 (2001), no. 2, 199226.

[25] P. Kirk and E. Klassen, Computing spectral flow via cup products. J. Differential Geom. 40 (1994), no. 3, 505-562.

[26] P. Kirk and M. Lesch, The $\eta$-invariant, Maslov index, and spectral flow for Dirac-type operators on manifolds with boundary. To appear in Forum Math., math.DG/0012123

[27] E. Klassen, Representations of knot groups in SU(2). Trans. Amer. Math. Soc. 326 (1991), no. 2, 795-828.

[28] H. B. Lawson and M. L. Michelsohn, Spin geometry. Princeton University Press, Princeton, 1989.

[29] M. LESCH and K. P. WoJCIEChOWSKI, On the $\eta$-invariant of generalized Atiyah-Patodi-Singer boundary value problems. Ill. J. Math. 40 (1996), 30-46

[30] C. Livingston, Knot theory. Carus Mathematical Monographs, 24. Mathematical Association of America, Washington, DC, 1993.

[31] W. Neumann, Homotopy invariance of Atiyah invariants. Algebraic and geometric topology (Proc. Sympos. Pure Math., Stanford Univ., Stanford, Calif., 1976), Part 2, pp. 181-188, Proc. Sympos. Pure Math., XXXII, Amer. Math. Soc., Providence, R.I., 1978.

[32] D. Quillen, Determinants of Cauchy-Riemann operators on Riemann surfaces. Functional Anal. Appl. 19 (1985), no. 1, 31-34.

[33] T. R. Ramadas, I. M. Singer, and J. Weitsman, Some comments on Chern-Simons gauge theory. Comm. Math. Phys. 126 (1989), no. 2, 409-420.

[34] D. Rolsen, Knots and links. Mathematics Lecture Series, No. 7. Publish or Perish, Inc., Berkeley, Calif., 1976.

[35] F. Waldhausen, On irreducible 3 -manifolds which are sufficiently large. Ann. of Math. (2) 87 (1968), 56-88.

[36] C. T. C. WALL, Non-additivity of the signature. Invent. Math. 7 (1969), 269274 . 
[37] C. T. C. WALL, Surgery on compact manifolds. Second edition. Edited and with a foreword by A. A. Ranicki. Mathematical Surveys and Monographs, 69. American Mathematical Society, Providence, RI, 1999.

[38] S. Weinberger, Homotopy invariance of $\eta$-invariants. Proc. Nat. Acad. Sci. U.S.A. 85 (1988), no. 15, 5362-5363.

Department of Mathematics, Indiana University

Bloomington, IN 47405, USA

and

Universität zu Köln, Mathematisches Institut

Weyertal 86-90, 50931 Köln, Germany

Email: pkirk@indiana.edu, lesch@mi.uni-koeln.de

URL: http://php.indiana.edu/ pkirk, http://www.mi.uni-koeln.de/ lesch

Received: 30 January 2003

Algebraic 63 Geometric Topology, Volume 3 (2003) 